\title{
Communication \\ The Evolution of Pleconaril: Modified O-Alkyl Linker Analogs Have Biological Activity towards Coxsackievirus B3 Nancy
}

\author{
Alexandrina Volobueva ${ }^{1,+}$, Anna Egorova ${ }^{2,+}$, Anastasia Galochkina ${ }^{1}$, Sean Ekins ${ }^{3}$, \\ Vladimir Zarubaev ${ }^{1}$ (D) and Vadim Makarov ${ }^{2, *}$ \\ 1 Saint-Petersburg Pasteur Institute, Mira str., 14, 197101 Saint Petersburg, Russia; \\ sasha-khrupina@mail.ru (A.V.); nastyagalochkina@yandex.ru (A.G.); zarubaev@gmail.com (V.Z.) \\ 2 Bach Institute of Biochemistry, Research Center of Biotechnology of the Russian Academy of Sciences, \\ Leninsky prospect, 33, build. 2, 119071 Moscow, Russia; anna.p.egorova@gmail.com \\ 3 Collaborations Pharmaceuticals, Inc., 840 Main Campus Drive, Lab 3510, Raleigh, NC 27606, USA; \\ sean@collaborationspharma.com \\ * Correspondence: makarov@inbi.ras.ru \\ + These authors contributed equally to this work.
}

Received: 10 February 2020; Accepted: 13 March 2020; Published: 16 March 2020

\begin{abstract}
Coxsackieviruses type B are one of the most common causes of mild upper respiratory and gastrointestinal illnesses. At the time of writing, there are no approved drugs for effective antiviral treatment for Coxsackieviruses type B. We used the core-structure of pleconaril, a well-known antienteroviral drug candidate, for the synthesis of novel compounds with O-propyl linker modifications. Some original compounds with 4 different linker patterns, such as sulfur atom, ester, amide, and piperazine, were synthesized according to five synthetic schemes. The cytotoxicity and bioactivity of 14 target compounds towards Coxsackievirus B3 Nancy were examined. Based on the results, the values of $50 \%$ cytotoxic dose $\left(\mathrm{CC}_{50}\right), 50 \%$ virus-inhibiting dose $\left(\mathrm{IC}_{50}\right)$, and selectivity index (SI) were calculated for each compound. Several of the novel synthesized derivatives exhibited a strong anti-CVB3 activity (SI $>20$ to $>200$ ). These results open up new possibilities for synthesis of further new selective anticoxsackievirus compounds.
\end{abstract}

Keywords: coxsackievirus; coxsackievirus B3 Nancy; viral myocarditis; antivirals; pleconaril

\section{Introduction}

Enteroviruses belonging to the Picornaviridae family are a diverse group of small (30-32 $\mathrm{nm}$ size) icosahedral non-enveloped viruses with single-stranded non-segmented positive RNA genome with a poly(A) tail. They are able to survive in harsh environments and can cause both self-limiting infections as well as polio, hand-foot-mouth disease, and heart and central nervous system diseases [1]. Currently, the genus Enterovirus encompasses 15 species: Enterovirus A-L and Rhinovirus A-C. Coxsackieviruses type B (CVBs) are members of Enterovirus B species and include six serotypes (CVB1-6). CVBs are lytic viruses but persistent infection responsible for chronic inflammation within target organs can be established. CVB3 often leads to mild upper respiratory and gastrointestinal illnesses, but it can also cause myocarditis [2,3]. Viral myocarditis is usually associated with dyspnea, arrhythmia, and chest pain and can lead to acute heart failure and sudden death. Currently available treatment is supportive and focuses on the symptomatic factors of disease [4,5].

To date, there are no approved antiviral agents for effective therapy of CVB3 infections. Currently the most advanced approaches for anti-CVB drug design are focused on the search for new direct antivirals, the modification of existing antiviral compounds, and drug repurposing screening [6]. 
Pleconaril, a well-known antienteroviral drug candidate with the capsid-binding mechanism of action, does not cover all of the Coxsackievirus B serotypes, including the typical representative, Coxsackievirus B3 Nancy, which is explored in this article [7-9].

Previously, we have reported that pleconaril resistance was overcome by unsubstituted analogues or by monosubstitution in the central phenyl ring [10]. In our most recent work, we showed the impact of the substitution pattern in the isoxazole and phenyl rings of the pleconaril core structure and their effect on antiviral activity [11]. The most active compound to date contains the 3- $\mathrm{N}, \mathrm{N}$-dimethylcarbamoyl group in the isoxazole ring and the 3-methyl group in the phenyl ring (Figure 1).

It is interesting to investigate how the replacement of the alkyl linker with different substituents affects the antienteroviral activity, because in the original pleconaril research project, G.D. Diana et al. varied the length of the aliphatic chain only $[12,13]$. Thus, in the present article, we have continued our investigation to study the influence of the pleconaril core structure and various modifications on the observed antiviral activity. We synthesized compounds with the general structure shown in Figure 1, which have the $\mathrm{N}, \mathrm{N}$-dimethylcarbamoyl or ethoxycarbonyl or methyl (like pleconaril) groups in the isoxazole ring and the 3-methyl group in phenyl ring, and then examined their inhibition activity against Coxsackievirus B3 Nancy.

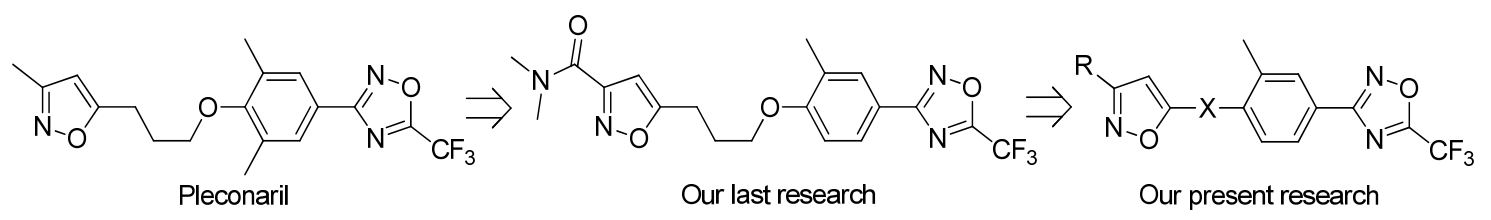

Figure 1. The Evolution of Pleconaril.

\section{Results and Discussion}

To explore the impact of the $O$-alkyl linker modification we, firstly, exchanged an oxygen atom for a sulfur atom with conservation of the 3-carbon chain; secondly, we introduced an ester or amide group into the linker; finally, we completely replaced the alkyl linker with piperazine. Syntheses of the compounds are presented in Schemes 1-4.

In the Scheme 1, the initial benzonitrile $\mathbf{1}$ was reacted with 5-chloro-1-pentyne in the presence of potassium carbonate and potassium iodide in NMP to produce pentynylthiobenzonitrile 2 . The reaction of $\mathbf{2}$ with excess of hydroxylamine hydrochloride and potassium carbonate in absolute refluxing ethanol provided amidoxime 3 with a yield of 92\%. Cyclization into 1,2,4-oxadiazole 4 was carried out using treatment of 3 with trifluoroacetic anhydride in pyridine. The cycloaddition of the S-pentyn-1 linker in 4 and 2-chloro-2-(hydroxyimino)acetic acid ethyl ester 5 or commercial acetaldoxime resulted in the target compounds $\mathbf{6 a}, \mathbf{b}$ with yields of $40 \%$ and $32 \%$, respectively. Finally, (carbethoxy-isoxazolyl)propyl)thio)phenyl)oxadiazole 6a reacted with dimethylamine solution in order to synthesize 7 with a yield of $57 \%$. 


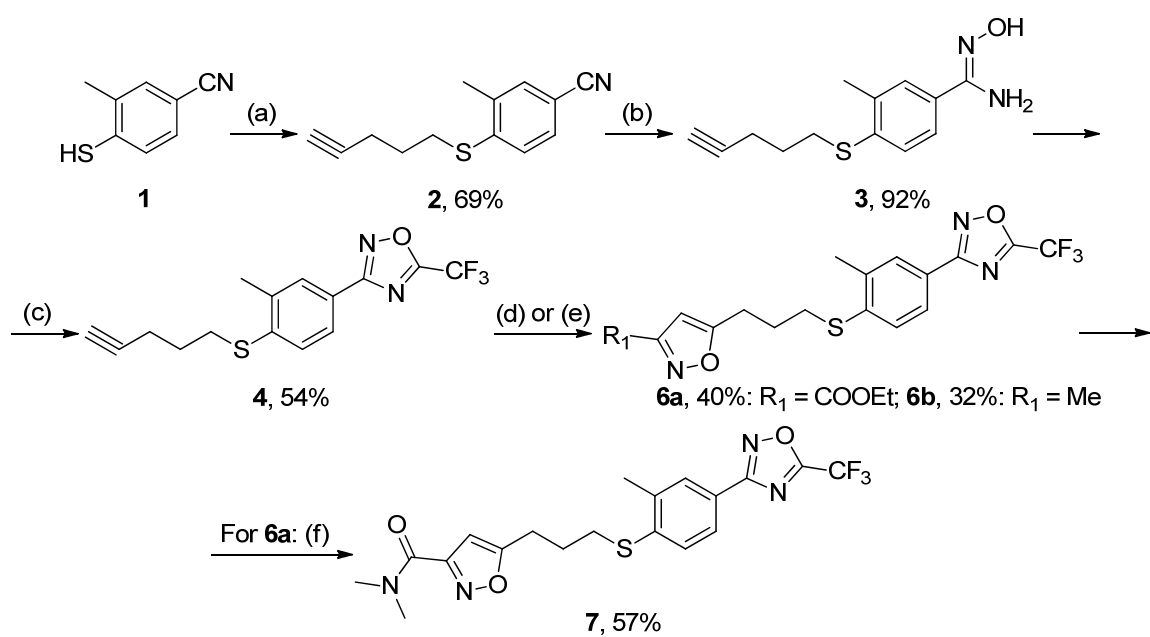

Scheme 1. Synthesis of 3-(3-methyl-4-((3-(3-substitutedisoxazol-5-yl)propyl)thio)phenyl)-5(trifluoromethyl)-1,2,4-oxadiazoles $\mathbf{6 a}, \mathbf{b}$ and 7. Reagents and conditions: (a) 5-chloro-1-pentyne, $\mathrm{K}_{2} \mathrm{CO}_{3}, \mathrm{KI}, \mathrm{NMP}, 65^{\circ} \mathrm{C}$; (b) $\mathrm{NH}_{2} \mathrm{OH} \cdot \mathrm{HCl}, \mathrm{K}_{2} \mathrm{CO}_{3}, \mathrm{EtOH}_{\text {abs }}$, reflux; (c) $\left(\mathrm{CF}_{3} \mathrm{CO}\right)_{2} \mathrm{O}$, pyridine, 80-90 ${ }^{\circ} \mathrm{C}$; (d) 2-chloro-2-(hydroxyimino)acetic acid ethyl ester 5, $\mathrm{Et}_{3} \mathrm{~N}, \mathrm{DMF}, 80-90{ }^{\circ} \mathrm{C}$; (e) acetaldoxime, $\mathrm{N}$-chlorosuccinimide, pyridine $\mathrm{cat}_{\text {cat }} \mathrm{Et}_{3} \mathrm{~N}, \mathrm{DMF}, 80-90{ }^{\circ} \mathrm{C}$; (f) dimethylamine solution 17 wt.\% in dioxane, $70-80^{\circ} \mathrm{C}$.

2-Methyl-4-(5-(trifluoromethyl)-1,2,4-oxadiazol-3-yl)phenyl ester of 3-(3-substituted isoxazol-5-yl)propionic acids 12a,b were synthesized according to Scheme 2. Because of the ester hydrolytic instability, synthesis of these compounds was started with formation of 1,2,4-oxadiazole cycle. Phenyloxadiazole $\mathbf{1 0}$ was successfully obtained via standard procedure (Scheme 1) from amidoxime 9 with a yield of $43 \%$. The Steglich esterification of phenol 10 with 4-pentynoic acid provided 11 in $72 \%$. In the final stage, treatment of pentynoate 11 by 2-chloro-2-(hydroxyimino)acetic acid ethyl ester $\mathbf{5}$ or acetaldoxime led to target compounds $\mathbf{1 2} \mathbf{a}, \mathbf{b}$ with low yields. Unfortunately, the reaction of 12a with non-water dimethylamine solution did not provide the corresponding product 13. In this case, the $\mathrm{C}-\mathrm{O}$ bond of the ester breaks rapidly, forming 10, for which the structure was confirmed by MS and NMR data.

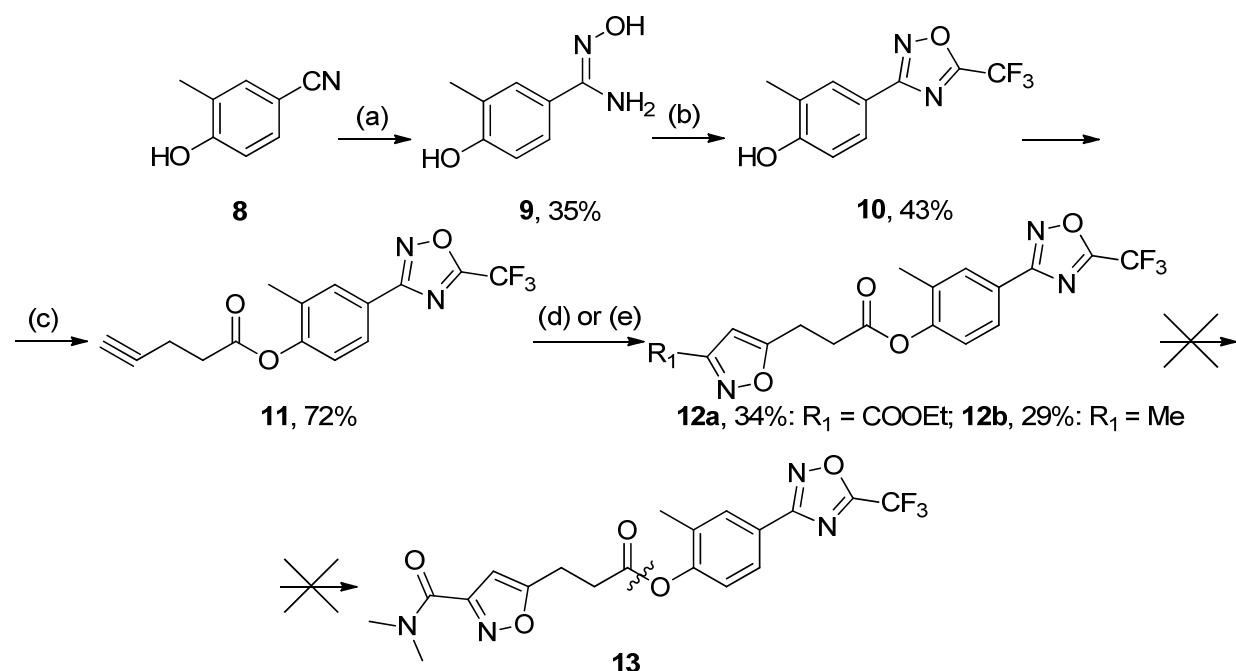

Scheme 2. Synthesis of 2-methyl-4-(5-(trifluoromethyl)-1,2,4-oxadiazol-3-yl)phenyl ester of 3-(3-substituted isoxazol-5-yl)propionic acids 12a,b. Reagents and conditions: (a) $\mathrm{NH}_{2} \mathrm{OH} \cdot \mathrm{HCl}$, $\mathrm{K}_{2} \mathrm{CO}_{3}, \mathrm{EtOH}_{\mathrm{abs}}$, reflux; (b) $\left(\mathrm{CF}_{3} \mathrm{CO}\right)_{2} \mathrm{O}$, pyridine, 80-90 ${ }^{\circ} \mathrm{C}$; (c) 4-pentynoic acid, DCC, pyridine, rt; (d) 2-chloro-2-(hydroxyimino)acetic acid ethyl ester 5, $\mathrm{Et}_{3} \mathrm{~N}, \mathrm{DMF}, 80-90{ }^{\circ} \mathrm{C}$; (e) acetaldoxime, $N$-chlorosuccinimide, pyridine ${ }_{\text {cat }}, \mathrm{Et}_{3} \mathrm{~N}, \mathrm{DMF}, 80-90{ }^{\circ} \mathrm{C}$. 
At first, we thought of using the same sequence of steps described above (Schemes 1 and 2) for the synthesis of 2-methyl-4-(5-(trifluoromethyl)-1,2,4-oxadiazol-3-yl)phenylamide of 3-(3-substituted isoxazol-5-yl)propionic acids $\mathbf{2 0 a , b}$ and 21, but this was not possible for two reasons. The first reason was asphaltization of the product as a result of the amidoxime formation reaction. Yet another reason was the reaction of trifluoroacetic anhydride with the free amino group of 4-amino- $N^{\prime}$-hydroxy-3-methylbenzimidamide at the second 1,2,4-oxadiazole cyclization stage. Therefore, another approach was designed.

$N$-Boc-protected aminobenzonitrile 15 was obtained from reaction of 4-amino-3-methylbenzonitrile 14 with $\mathrm{Boc}_{2} \mathrm{O}$ in non-solvent conditions with a yield of $93 \%$. The consistent treatment of 15 with hydroxylamine hydrochloride and trifluoroacetic anhydride provided oxadiazole 17. For selective cleavage of the $N$-Boc group, compound 17 was treated by trifluoroacetic acid to give aniline 18 with a yield of $99 \%$. The reaction of 18 with 4-pentynoic acid in the presence of EDCl as dehydrating agent, DMAP as catalyst in the medium of DCM provided pentynamide 19 . Final compounds $20 \mathbf{a}, \mathbf{b}$ were synthesized as described above (Schemes 1 and 2). Unlike the ester (Scheme 2), the amide group is more hydrolytically stable. Treatment of 20a with dimethylamine solution prepared the corresponding product 21 with a yield of $63 \%$.

Two different ways for the synthesis of piperazine derivatives were developed as indicated in Schemes 4 and 5. The initial Scheme 4 was designed based on intermediate 22, which was coupled with two isoxazoles, and in the final stages, 1,2,4-oxadiazole formation was provided. Scheme 5 was developed in order to improve Scheme 4, and in this case, the synthesis of target piperazine derivatives was dependent on the key intermediate, compound 34, which was especially prepared for these goals.

The couplings of piperazinylbenzonitrile 22 and isoxazoles $\mathbf{2 3}$ or $\mathbf{2 4}$ in the presence of potassium carbonate in refluxing acetonitrile give the corresponding compounds $\mathbf{2 5 a}, \mathbf{b}$ with yields of $40 \%$ and $56 \%$, respectively. In the case of derivative $25 \mathbf{a}$, having the carbethoxy group in the isoxazole, it was not possible to carry out the reaction of amidoxime formation directly, in our opinion, due to hydrolysis of the ester. Therefore, the ester group was replaced with the amide group using dimethylamine solution to provide $\mathbf{2 6}$. In the case of $\mathbf{2 5 b}$, direct reaction with hydroxylamine hydrochloride successfully provided amidoxime $\mathbf{2 7 b}$. Finally, oxadiazole cyclization with the formation of piperazine derivatives $\mathbf{2 8 a} \mathbf{b}$ took place under typical conditions (Schemes 1-3). 


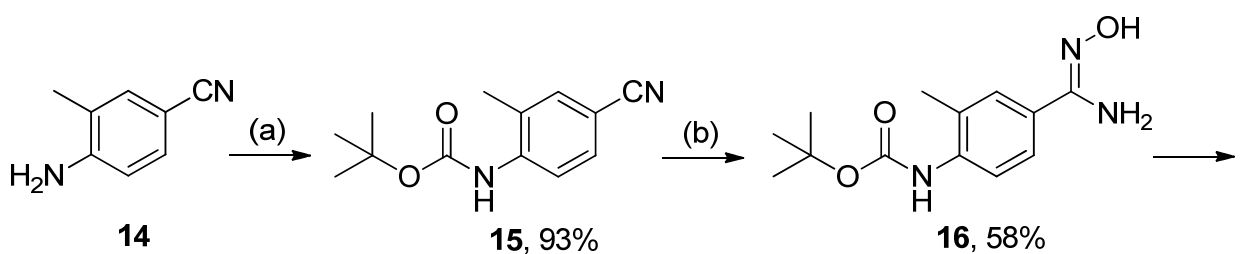

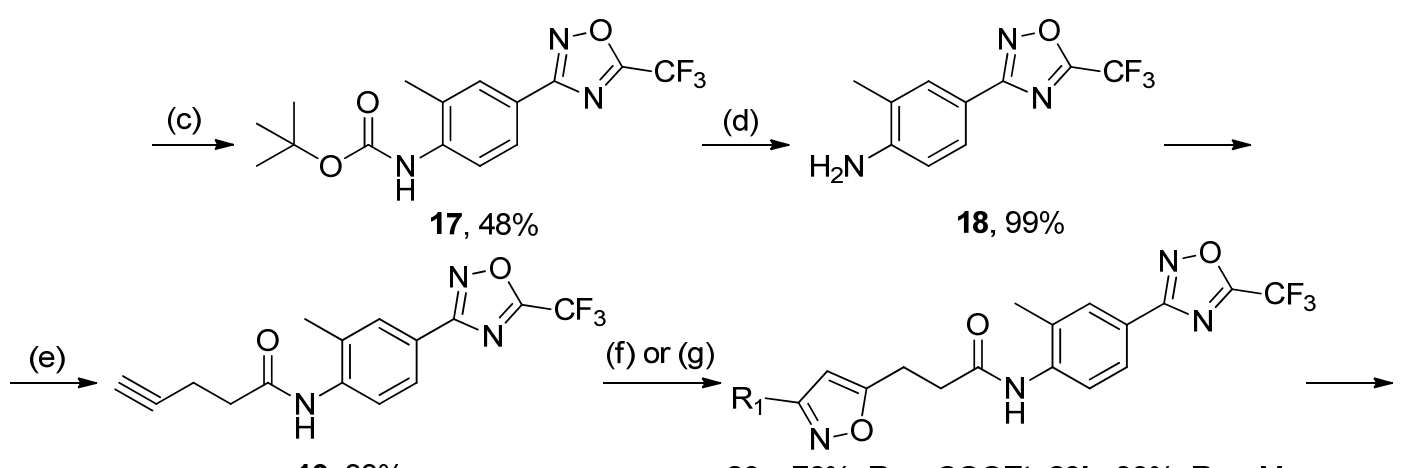

$19,38 \%$

20a, $78 \%: \mathrm{R}_{1}=$ COOEt; $20 \mathrm{~b}, 83 \%: \mathrm{R}_{1}=\mathrm{Me}$<smiles>CC#CCN(C)C(=O)c1cc(CCC(=O)Nc2ccc(-c3noc(C(F)(F)F)n3)cc2C)on1</smiles>

Scheme 3. Synthesis of 2-methyl-4-(5-(trifluoromethyl)-1,2,4-oxadiazol-3-yl)phenylamide of 3-(3-substituted isoxazol-5-yl)propionic acids 20a,b and 21. Reagents and conditions: (a) $\mathrm{Boc}_{2} \mathrm{O}$, $85^{\circ} \mathrm{C}$; (b) $\mathrm{NH}_{2} \mathrm{OH} \cdot \mathrm{HCl}, \mathrm{K}_{2} \mathrm{CO}_{3}, \mathrm{EtOH}_{\text {abs }}$, reflux; (c) $\left(\mathrm{CF}_{3} \mathrm{CO}\right)_{2} \mathrm{O}$, pyridine, $80-90{ }^{\circ} \mathrm{C}$; (d) $\mathrm{CF}_{3} \mathrm{COOH}$, DCM, rt; (e) 4-pentynoic acid, EDCl, DMAP, DCM, rt; (f) 2-chloro-2-(hydroxyimino)acetic acid ethyl ester 5, $\mathrm{Et}_{3} \mathrm{~N}, \mathrm{DMF}, 80-90^{\circ} \mathrm{C}$; (g) acetaldoxime, $\mathrm{N}$-chlorosuccinimide, pyridine cat, $\mathrm{Et}_{3} \mathrm{~N}$, DMF, 80-90 ${ }^{\circ} \mathrm{C}$; (h) dimethylamine solution $17 \mathrm{wt} . \%$ in dioxane, $70-80^{\circ} \mathrm{C}$.

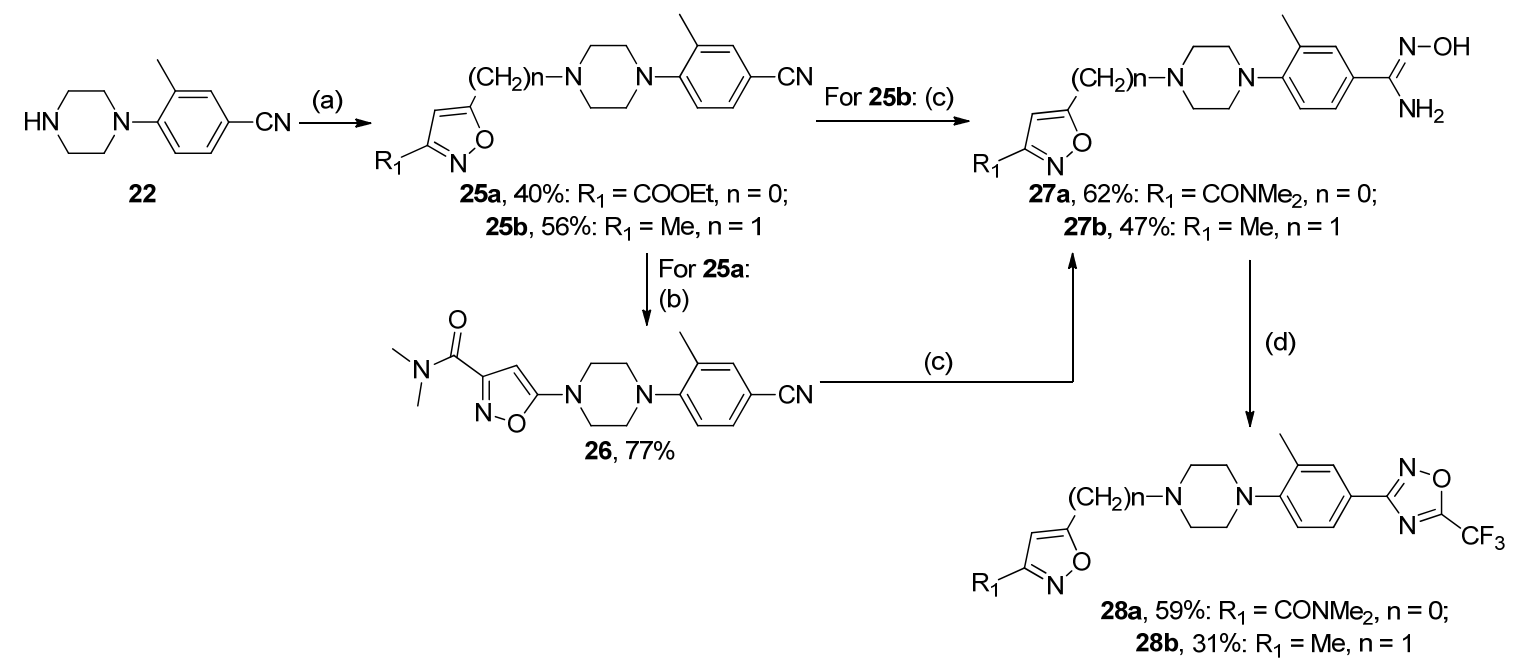

Scheme 4. Synthesis of piperazine derivatives $\mathbf{2 8 a}$, b. (a) ethyl 5-chloroisoxazole-3-carboxylate 23 or 5-(chloromethyl)-3-methylisoxazole $24, \mathrm{~K}_{2} \mathrm{CO}_{3}$, acetonitrile, reflux; (b) dimethylamine solution 33 wt. $\%$ in $\mathrm{H}_{2} \mathrm{O}, \mathrm{EtOH}$; (c) $\mathrm{NH}_{2} \mathrm{OH} \cdot \mathrm{HCl}, \mathrm{K}_{2} \mathrm{CO}_{3}, \mathrm{EtOH}_{\mathrm{abs}}$, reflux; (d) $\left(\mathrm{CF}_{3} \mathrm{CO}\right)_{2} \mathrm{O}$, pyridine, $85^{\circ} \mathrm{C}$.

In another approach, 1-(4-bromo-2-methylphenyl)piperazine 29 was $N$-protected with acetic anhydride to yield $\mathbf{3 0}$. The bromine atom in $\mathbf{3 0}$ was exchanged to a cyano group by copper(I) cyanide in NMP at high temperature in accordance with the procedure [14]. Benzonitrile 31 was treated in two steps under typical conditions for 1,2,4-oxadiazole cyclization (Schemes 1-4) to provide compound 33 
with a yield of $62 \%$. For selective cleavage of the acetyl group, 33 was worked up with hydrochloric acid in ethanol to give ((piperazinyl)phenyl)oxadiazole 34 in $65 \%$. While working on piperazine analogue synthesis, we came across an article in which compounds with similar structural fragments like ours (the phenyl ring is bound to piperazine, which through a carbonyl group is bound to isoxazole) were studied against influenza virus A [15]. It was interesting to investigate this structural fragment in the skeleton of our compound. For this purpose, compound 34 was coupled with commercially available 3-phenylisoxazole-5-carboxylic acid or 5-methyl-3-phenylisoxazole-4-carboxylic acid in the presence of EDCl, DMAP, and DCM to obtain the products $35 a, b$ with yields of $44 \%$ and $38 \%$, respectively. Moreover, 34 was reacted with isoxazole 23 to provided $28 \mathrm{c}$ in $80 \%$. Finally, 34 was successively treated with propargyl bromide and 2-chloro-2-(hydroxyimino)acetic acid methyl ester 36 to give $\mathbf{2 8 d}$. The structures of the all derivatives were characterized by ${ }^{1} \mathrm{H}-\mathrm{NMR}$ spectroscopy and mass spectrometry.

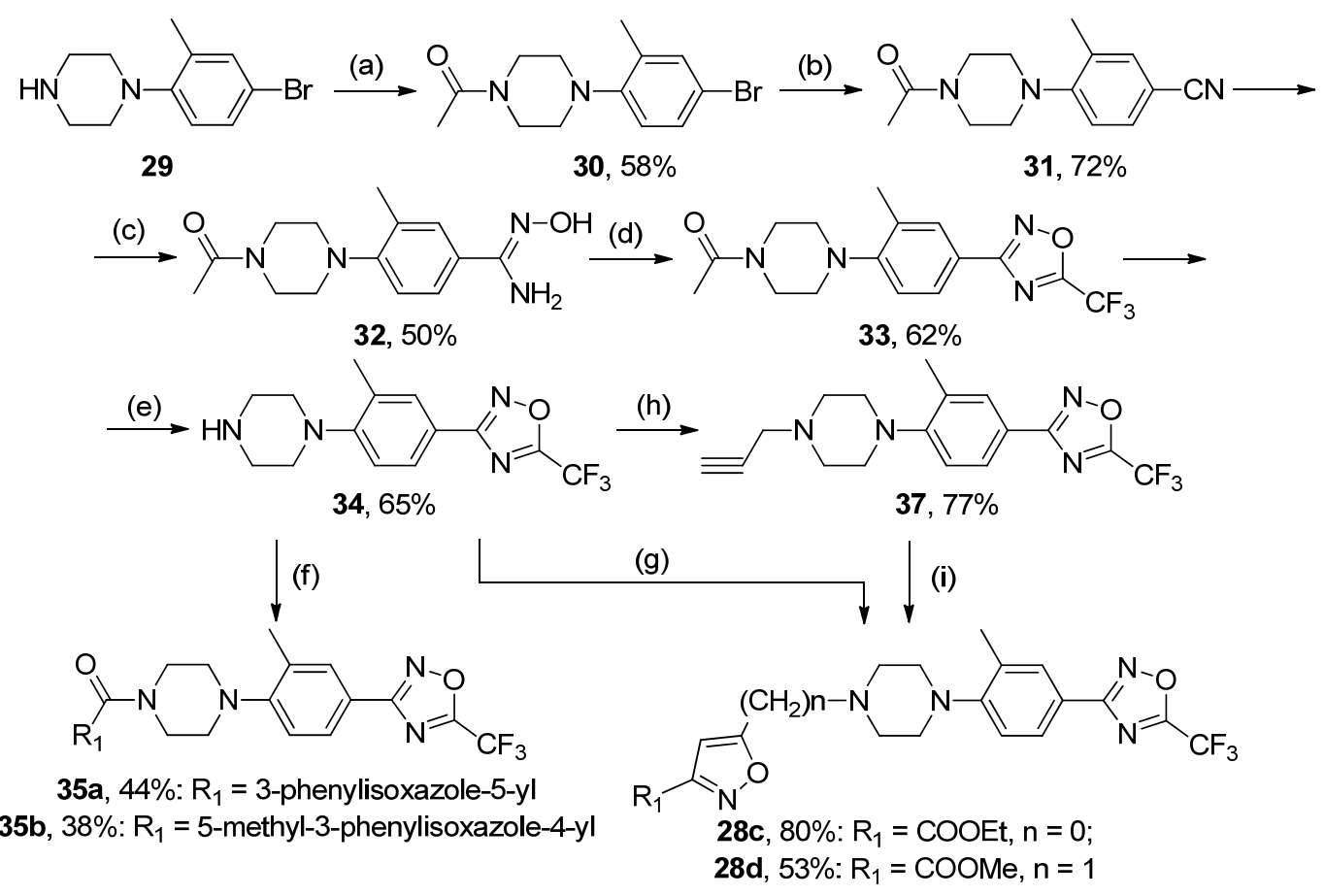

Scheme 5. Synthesis of piperazine derivatives $35 \mathbf{a}, \mathbf{b}, \mathbf{2 8 c}$, d. (a) $\mathrm{Ac}_{2} \mathrm{O}, 65^{\circ} \mathrm{C}$; (b) $\mathrm{CuCN}, \mathrm{NMP}$, $150{ }^{\circ} \mathrm{C}$; (c) $\mathrm{NH}_{2} \mathrm{OH} \cdot \mathrm{HCl}, \mathrm{K}_{2} \mathrm{CO}_{3}, \mathrm{EtOH}_{\mathrm{abs}}$, reflux; (d) $\left(\mathrm{CF}_{3} \mathrm{CO}\right)_{2} \mathrm{O}$, pyridine, $80-90^{\circ} \mathrm{C}$; (e) $\mathrm{HCl}, \mathrm{EtOH}$; (f) 3-phenylisoxazole-5-carboxylic acid or 5-methyl-3-phenylisoxazole-4-carboxylic acid, EDC, DMAP, DCM, rt; (g) 5-chloroisoxazole-3-carboxylate $23, \mathrm{~K}_{2} \mathrm{CO}_{3}$, acetonitrile, reflux; (h) propargyl bromide, $\mathrm{K}_{2} \mathrm{CO}_{3}, \mathrm{KI}$, acetonitrile, $50{ }^{\circ} \mathrm{C}$; (i) 2-chloro-2-(hydroxyimino)acetic acid methyl ester $36, \mathrm{Et}_{3} \mathrm{~N}$, DMF, $80-90{ }^{\circ} \mathrm{C}$.

The final compounds $\mathbf{6 a}, \mathbf{b}, \mathbf{7}, \mathbf{1 2 a}, \mathbf{b}, \mathbf{2 0 a}, \mathbf{b}, \mathbf{2 1}, \mathbf{2 8 a}, \mathbf{b}, \mathbf{3 5 a}, \mathbf{b}$, and $\mathbf{2 8 c}$, d were tested for their activity against Coxsackievirus B3 Nancy in the viral yield reduction assay. Derivatives $\mathbf{3 5 a} \mathbf{a} \mathbf{b}$ were also tested for anti-influenza A activity. Based on the results obtained, 50\% cytotoxic concentration $\left(\mathrm{CC}_{50}\right)$ and $50 \%$ inhibiting concentration $\left(\mathrm{IC}_{50}\right)$ were calculated for each compound. The selectivity index was calculated for each compound as a ratio of $\mathrm{CC}_{50}$ to $\mathrm{IC}_{50}$. The biological results are summarized in Tables 1 and 2 below. Pleconaril was used for comparison. 
Table 1. Antiviral activity of synthesized compounds towards coxsackievirus B3 strain Nancy.

\begin{tabular}{|c|c|c|c|c|c|c|}
\hline № & $\mathbf{R}_{1}$ & $\mathrm{X}$ & $n$ & $\mathrm{CC}_{50}, \mu \mathrm{M}$ & $\begin{array}{c}\mathrm{IC}_{50} \\
\text { towards } \\
\text { CVB3, } \mu \mathrm{M}\end{array}$ & SI \\
\hline $6 a$ & COOEt & $S$ & 3 & $484.8 \pm 20.1$ & $21.0 \pm 1.5$ & 23 \\
\hline $6 \mathrm{~b}$ & $\mathrm{Me}$ & $\mathrm{S}$ & 3 & $>2608.3$ & $15.6 \pm 1.4$ & $>167$ \\
\hline 7 & $\mathrm{CONMe}_{2}$ & $\mathrm{~S}$ & 3 & $>2270.45$ & $18.6 \pm 1.3$ & $>122$ \\
\hline $12 \mathrm{a}$ & COOEt & $\mathrm{COO}$ & 2 & $682.8 \pm 30.4$ & $9.1 \pm 0.8$ & 75 \\
\hline $12 b$ & $\mathrm{Me}$ & $\mathrm{COO}$ & 2 & $464.2 \pm 30.3$ & $13.1 \pm 0.9$ & 35 \\
\hline $20 \mathrm{a}$ & COOEt & $\mathrm{CONH}$ & 2 & $1724.6 \pm 70.5$ & $18.2 \pm 1.6$ & 95 \\
\hline $20 \mathrm{~b}$ & $\mathrm{Me}$ & $\mathrm{CONH}$ & 2 & $1743.2 \pm 120.2$ & $76.2 \pm 5.3$ & 23 \\
\hline 21 & $\mathrm{CONMe}_{2}$ & $\mathrm{CONH}$ & 2 & $1689.6 \pm 70.7$ & 6.8 & 248 \\
\hline $28 \mathrm{a}$ & $\mathrm{CONMe}_{2}$ & piperazine & 0 & $1867.1 \pm 90.4$ & $9.1 \pm 0.7$ & 205 \\
\hline $28 \mathrm{~b}$ & $\mathrm{Me}$ & piperazine & 1 & $2455.2 \pm 170.5$ & $41.7 \pm 2.1$ & 59 \\
\hline $28 \mathrm{c}$ & COOEt & piperazine & 0 & $53.2 \pm 4.5$ & $48.7 \pm 4.4$ & 1 \\
\hline $28 \mathrm{~d}$ & COOMe & piperazine & 1 & $203.8 \pm 14.7$ & $3.9 \pm 0.3$ & 52 \\
\hline $35 \mathrm{a}$ & 3-phenylisoxazole-5-carbonyl & piperazine & 0 & $1522.4 \pm 70.3$ & $>620.5$ & $<2$ \\
\hline \multirow[t]{2}{*}{$35 b$} & 5-methyl-3-phenylisoxazole-4-carbonyl & piperazine & 0 & $2010.1 \pm 120.8$ & $16.1 \pm 1.1$ & 125 \\
\hline & Pleconaril & & & $2396.7 \pm 160.2$ & $18.4 \pm 1.5$ & 130 \\
\hline
\end{tabular}

Table 2. Antiviral activity of compounds 35a,b towards influenza virus A/Puerto Rico/8/34 (H1N1).

\begin{tabular}{cccc}
\hline № & $\mathbf{C C}_{\mathbf{5 0}}, \boldsymbol{\mu M}$ & IC $_{\mathbf{5 0}}$ Towards IAV, $\boldsymbol{\mu M}$ & SI \\
\hline 35a & $1449.3 \pm 102.1$ & $>492.8$ & $<3$ \\
35b & $>603.6$ & $>603.6$ & n/a ${ }^{1}$ \\
\hline \multicolumn{4}{c}{${ }^{1}$ n/a-not applicable. }
\end{tabular}

A large majority of the tested compounds (85\%) were non-toxic and demonstrated $\mathrm{CC}_{50}>400 \mu \mathrm{M}$, except for $\mathbf{2 8 c}$ and $\mathbf{2 8 d}$, which have $\mathrm{CC}_{50}$ values about 53.2 and $203.8 \mu \mathrm{M}$, respectively. Replacement of an oxygen atom with a sulfur atom in the propyl linker led to a decrease in the activity. Derivatives with the $S$-linker $\mathbf{6 a}, \mathbf{6} \mathbf{b}$, and $\mathbf{7}$, having $\mathrm{COOEt}, \mathrm{Me}$, and $\mathrm{CONMe}_{2}$ groups in the isoxazole ring, respectively, demonstrated $\mathrm{IC}_{50}$ values of 21.0, 15.6, and $18.6 \mu \mathrm{M}$, while derivatives with the O-linker, having the same groups in the same position, showed $\mathrm{IC}_{50}$ about 18.96 [11], 4.6 [10], and $2.76 \mu \mathrm{M}$ [11], respectively.

Insertion of the carbonyl group to the $O$-linker exhibited dissimilar results: in the case of the isoxazole COOEt group the modification increased the anti-CVB3 activity $(9.1 \mu \mathrm{M}$ for $\mathrm{C}(\mathrm{O}) \mathrm{O}$-linker and $18.96 \mu \mathrm{M}$ for $O$-linker), but for the methyl group, the variation greatly reduced the ability to inhibit CVB3 replication $(13.1 \mu \mathrm{M}$ for $\mathrm{C}(\mathrm{O}) \mathrm{O}$-linker and $4.6 \mu \mathrm{M}$ for $O$-linker).

When the carbamoyl group was introduced instead of the $O$-linker $(\mathbf{2 0 a}, \mathbf{b})$, the antiviral activity was reduced again. However, for compound 21, this change was reflected positively: this derivative has a good $\mathrm{IC}_{50}(6.8 \mu \mathrm{M})$ and, subsequently, the highest selectivity index (248) in this series.

Finally, we examined the impact of the propyl linker substitution to piperazine on viral inhibition. Derivatives without 1-carbon chain between piperazine and isoxazole cycles, i.e., piperazine linked to isoxazole directly, having the dimethylcarbamoyl group $28 \mathrm{a}$, are more active $(\mathrm{SI}=205)$ than the same derivative with the carbethoxy group $28 \mathrm{c}(\mathrm{SI}=1)$. On the other hand, antiviral testing of derivatives with 1-carbon chain $\mathbf{2 8 b}$ and $\mathbf{2 8 d}$ exhibited inconclusive results: $\mathbf{2 8 b}$ has a poor $\mathrm{IC}_{50}$ and good $\mathrm{CC}_{50}$ values, whereas $28 \mathrm{~d}$ showed the highest value of $\mathrm{IC}_{50}$ in the series, but unfortunately 28d was cytotoxic $\left(\mathrm{CC}_{50} \sim 203.8 \mu \mathrm{M}\right)$. Derivative $35 \mathrm{~b}$ with $\mathrm{R}_{1}=5$-methyl-3-phenylisoxazole-4-yl has a selectivity index like the reference compound ( $\mathrm{SI}=125$ for $35 \mathrm{~b}$ and 130 for pleconaril) and derivative 35a with $R_{1}=3$-phenylisoxazole-5-yl was inactive towards CVB3. Both of the compounds were inactive against the influenza virus A (see Table 2). 
The curves demonstrating the cytotoxic and virus-inhibiting properties of the most potent compounds in the series-6b, 21, 28a-are shown in Figure 2.

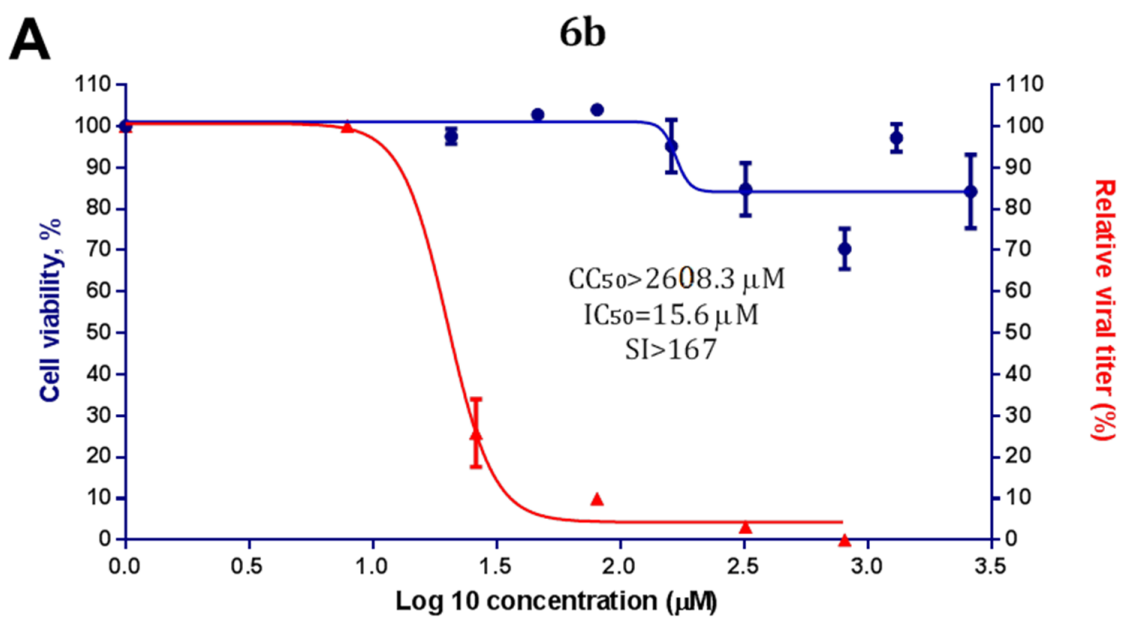

$\rightarrow$ Cell viability $(\%) \quad \neg$ Relative viral titer (\%)

B

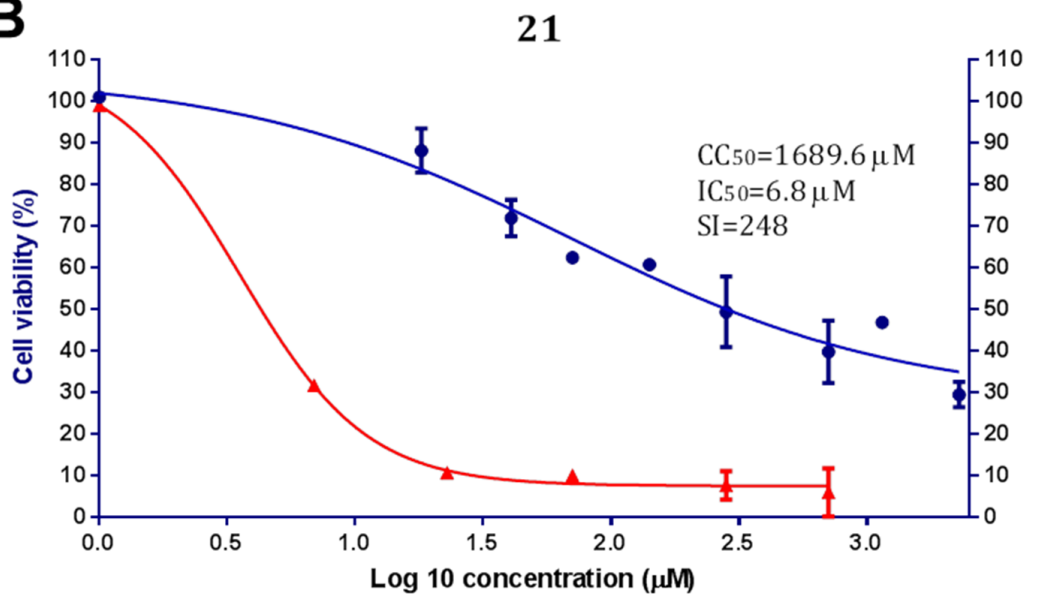

离

Cell viability (\%)

$\dashv$ Relative viral titer (\%)

C

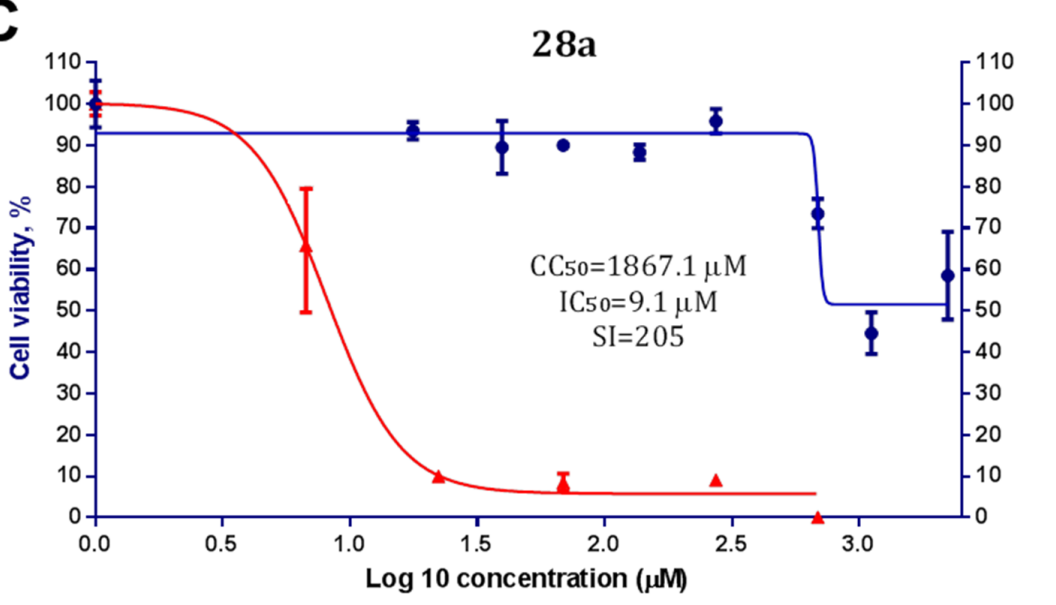

Cell viability (\%)

$\mp$ Relative viral titer (\%)

Figure 2. Cytotoxicity and anticoxsackievirus B3 activity of $\mathbf{6 b}(\mathrm{A}), \mathbf{2 1}(\mathrm{B})$, and $\mathbf{2 8 a}(\mathrm{C})$ : logistic curves for cell viability (MTT assay) and relative viral titer (virus yield reduction assay). 


\section{Materials and Methods}

\subsection{General Information}

All reagents and solvents were purchased from commercial suppliers and used without further purification. ${ }^{1} \mathrm{H}$ and ${ }^{13} \mathrm{C}$ spectra were measured on a Bruker AC-300 $\left(300 \mathrm{MHz},{ }^{1} \mathrm{H}\right)$ or Bruker AC-200 $\left(50 \mathrm{MHz},{ }^{13} \mathrm{C}\right)$. Chemical shifts were measured in $\mathrm{DMSO}-\mathrm{d}_{6}$ or $\mathrm{CDCl}_{3}$, using tetramethylsilane as an internal standard. The following abbreviations are used to indicate the multiplicity: s, singlet; $\mathrm{d}$, doublet; $\mathrm{t}$, triplet; q, quartet; quin, quintet; $\mathrm{m}$, multiplet; dd, doublet of doublets; td, triplet of doublets; dt, doublet of triplets; ddd, doublet of doublet of doublets; bs, broad signal. Mass spectra were obtained on a Finnigan SSQ-700 with direct injection. A Waters Micromass ZQ detector was used in EI MS for identification of various products. Melting points were determined on Electrothermal 9001 (10 ${ }^{\circ} \mathrm{C}$ per min) and are uncorrected. Merck silica gel 60 F254 plates were used for analytical TLC; column chromatography was performed on Merck silica gel 60 (70-230 mesh).

4-mercapto-3-methylbenzonitrile 1 was obtained by the Newman-Kwart rearrangement from commercially available 4-hydroxy-3-methylbenzonitrile in three steps [16]. 4-hydroxy-3-methylbenzonitrile 8 was synthesized from corresponding phenol by $N$-bromosuccinimide (NBS) bromination and subsequent change bromine atom to cyano group by copper (I) cyanide in dry dimethylformamide [14,17]. 4-amino-3-methylbenzonitrile 14 was obtained by the method in the patent [18]. 3-methyl-4-(piperazin-1-yl)benzonitrile 22 was synthesized from 4-amino-3-methylbenzonitrile 14 and bis(2-chloroethyl)amine [19]. 1-(4-Bromo-2-methylphenyl)piperazine 29 was synthesized by the method in the article by Ge Z. et al. [20]. 2-Chloro-2-(hydroxyimino)acetic acid ethyl ester $\mathbf{5}$ or 2-chloro-2-(hydroxyimino)acetic acid methyl ester 36 were synthesized from the corresponding glycine ester hydrochloride by nitrosation with sodium nitrite and hydrochloric acid [21]. Ethyl 5-chloroisoxazole-3-carboxylate 23 was obtained by the procedure in [22]. 5-(Chloromethyl)-3-methylisoxazole 24 was synthesized according to Li W.-T. et al. [23].

\subsection{Synthesis}

\subsubsection{Synthesis of 3-Methyl-4-(pent-4-yn-1-ylthio)benzonitrile 2}

A mixture of 4-mercapto-3-methylbenzonitrile 1 ( $1 \mathrm{~mol}$ ), finely divided $\mathrm{K}_{2} \mathrm{CO}_{3}(5 \mathrm{~mol}), \mathrm{KI}(0.01$ mol), 5-chloro-1-pentyne ( $1.5 \mathrm{~mol})$, and $\mathrm{N}$-methylpyrrolidone- 2 was heated at $65^{\circ} \mathrm{C}$ for $24 \mathrm{~h}$. The cooled reaction mixture was treated by cold water and stirred for 3-4 h. The solid was collected and recrystallized from methanol. Light beige solid, yield $69 \%$, m.p. $36-38{ }^{\circ} \mathrm{C}$. MS (EI), $m / z\left(I_{\text {relat }} .(\%)\right): 215$ $[\mathrm{M}]^{+}$(67). Calc. 215.3140, $\mathrm{C}_{13} \mathrm{H}_{13} \mathrm{NS} .{ }^{1} \mathrm{H}-\mathrm{NMR}$ (DMSO-d $)_{6}$ : $\delta 1.94\left(2 \mathrm{H}\right.$, quint, $\left.J=7.3, \mathrm{CH}_{2} \mathrm{CH}_{2} \mathrm{CH}_{2} \mathrm{~S}\right)$, $2.25\left(3 \mathrm{H}, \mathrm{s}, \mathrm{CH}_{3}\right), 2.30\left(2 \mathrm{H}, \mathrm{m}, \mathrm{CH}_{2} \mathrm{CH}_{2} \mathrm{CH}_{2} \mathrm{~S}\right), 2.77\left(1 \mathrm{H}, \mathrm{s}, \mathrm{CHCCH}_{2}\right), 3.15\left(2 \mathrm{H}, \mathrm{t}, J=7.3, \mathrm{CH}_{2} \mathrm{CH}_{2} \mathrm{CH}_{2} \mathrm{~S}\right)$, $7.46(1 \mathrm{H}, \mathrm{d}, J=8.8, \mathrm{H} 6), 7.62(1 \mathrm{H}, \mathrm{dd}, J=8.8, J=0.5, \mathrm{H} 5), 7.63(1 \mathrm{H}, \mathrm{s}, \mathrm{H} 3) \mathrm{ppm}$.

\subsubsection{General Procedure for the Synthesis of Compounds 3, 9, 16, 27a, b, 32}

A mixture of benzonitriles $2, \mathbf{8}, \mathbf{1 5}, \mathbf{2 5 b}, \mathbf{2 6}$, and 31 (1 mmol), finely divided $\mathrm{K}_{2} \mathrm{CO}_{3}(5 \mathrm{mmol})$, and hydroxylamine hydrochloride $(5 \mathrm{mmol})$ in absolute ethanol was refluxed for $24 \mathrm{~h}$. The hot reaction mixture was filtered, and the remaining solids were washed with hot acetone. The combined filtrates were concentrated in vacuo. The residue was recrystallized from the corresponding solvent (in parentheses following mp data).

$N^{\prime}$-Hydroxy-3-methyl-4-(pent-4-yn-1-ylthio)benzimidamide 3, Light yellow solid, yield 92\%, m.p. $64-66{ }^{\circ} \mathrm{C}$ (EtOH). MS (EI), $m / z$ (I relat. $(\%)): 248[\mathrm{M}]^{+}(54)$. Calc. $248.3439, \mathrm{C}_{13} \mathrm{H}_{16} \mathrm{~N}_{2} \mathrm{OS} .{ }^{1} \mathrm{H}-\mathrm{NMR}\left(\mathrm{DMSO}-d_{6}\right): \delta$ $1.86\left(2 \mathrm{H}\right.$, quint, $\left.J=7.3, \mathrm{CH}_{2} \mathrm{CH}_{2} \mathrm{CH}_{2} \mathrm{~S}\right), 2.37\left(2 \mathrm{H}, \mathrm{t}, \mathrm{J}=7.3, \mathrm{CH}_{2} \mathrm{CH}_{2} \mathrm{CH}_{2} \mathrm{~S}\right), 2.42\left(3 \mathrm{H}, \mathrm{s}, \mathrm{CH}_{3} \mathrm{Ph}\right), 2.77$ $\left(1 \mathrm{H}, \mathrm{s}, \mathrm{CHCCH}_{2}\right), 3.25\left(2 \mathrm{H}, \mathrm{t}, J=7.3, \mathrm{CH}_{2} \mathrm{CH}_{2} \mathrm{CH}_{2} \mathrm{~S}\right), 4.96(1 \mathrm{H}, \mathrm{s}, \mathrm{NOH}), 5.05\left(2 \mathrm{H}, \mathrm{brs}, \mathrm{NH}_{2}\right), 7.13(1 \mathrm{H}$, $\mathrm{d}, J=8.8, \mathrm{H} 6), 7.29(1 \mathrm{H}, \mathrm{dd}, J=8.8, J=0.5, \mathrm{H} 5), 7.33(1 \mathrm{H}, \mathrm{s}, \mathrm{H} 3) \mathrm{ppm}$. 
$\mathrm{N}^{\prime}$,4-dihydroxy-3-methylbenzimidamide 9, Light beige solid, yield 35\%, m.p. $70-71{ }^{\circ} \mathrm{C}$ (EtOH). MS (EI), $m / z\left(I_{\text {relat }} .(\%)\right): 166[\mathrm{M}]^{+}(100)$. Calc. $166.1772, \mathrm{C}_{8} \mathrm{H}_{10} \mathrm{~N}_{2} \mathrm{O}_{2} \cdot{ }^{1} \mathrm{H}-\mathrm{NMR}$ (DMSO- $\left.d_{6}\right): \delta 2.30\left(3 \mathrm{H}, \mathrm{s}, \mathrm{CH}_{3} \mathrm{Ph}\right)$, $4.99(1 \mathrm{H}, \mathrm{s}, \mathrm{NOH}), 5.09\left(2 \mathrm{H}, \mathrm{brs}, \mathrm{NH}_{2}\right), 6.83(1 \mathrm{H}, \mathrm{d}, J=7.5, \mathrm{H} 6), 7.34(1 \mathrm{H}, \mathrm{d}, J=7.5, \mathrm{H} 5), 7.51(1 \mathrm{H}, \mathrm{s}$, H3) ppm.

Tert-butyl (4-(N'-hydroxycarbamimidoyl)-2-methylphenyl)carbamate 16, White solid, yield 58\%, m.p. $74-76{ }^{\circ} \mathrm{C}$ (decomp.) (iPrOH). MS (EI), $m / z\left(I_{\text {relat }} .(\%)\right): 265[\mathrm{M}]^{+}(83)$. Calc. $265.3083, \mathrm{C}_{13} \mathrm{H}_{19} \mathrm{~N}_{3} \mathrm{O}_{3}$. ${ }^{1} \mathrm{H}-\mathrm{NMR}$ (DMSO-d $\left.d_{6}\right): \delta 1.43(9 \mathrm{H}, \mathrm{s}, \mathrm{tBu}), 2.22\left(3 \mathrm{H}, \mathrm{s}, \mathrm{CH}_{3} \mathrm{Ph}\right), 4.94(1 \mathrm{H}, \mathrm{s}, \mathrm{NOH}), 5.03\left(2 \mathrm{H}, \mathrm{brs}, \mathrm{NH}_{2}\right)$, $6.81(1 \mathrm{H}, \mathrm{d}, J=7.5, \mathrm{H} 6), 7.24(1 \mathrm{H}, \mathrm{d}, J=7.5, \mathrm{H} 5), 7.33(1 \mathrm{H}, \mathrm{s}, \mathrm{H} 3) \mathrm{ppm}$.

5-(4-(4-(N'-Hydroxycarbamimidoyl)-2-methylphenyl)piperazin-1-yl)-N,N-dimethylisoxazole-3-carboxamide 27a, White solid, yield $62 \%, \mathrm{mp} 203-205^{\circ} \mathrm{C}(\mathrm{EtOH})$. MS (EI), $\left.\mathrm{m} / z\left(I_{\text {relat }} . \%\right)\right): 372[\mathrm{M}]^{+}(67)$. Calc. 372.4216, $\mathrm{C}_{18} \mathrm{H}_{24} \mathrm{~N}_{6} \mathrm{O}_{3} .{ }^{1} \mathrm{H}-\mathrm{NMR}$ (DMSO-d $\left.d_{6}\right): \delta 2.22\left(3 \mathrm{H}, \mathrm{s}, \mathrm{CH}_{3} \mathrm{Ph}\right), 2.73\left(6 \mathrm{H}, \mathrm{s}, \mathrm{N}\left(\mathrm{CH}_{3}\right)_{2}\right), 3.24(4 \mathrm{H}, \mathrm{brt}$, $\left.\mathrm{N}\left(\mathrm{CH}_{2}\right)_{2}\right), 3.21\left(4 \mathrm{H}, \mathrm{brt}, \mathrm{N}\left(\mathrm{CH}_{2}\right)_{2}\right), 4.94(1 \mathrm{H}, \mathrm{s}, \mathrm{NOH}), 5.01\left(2 \mathrm{H}, \mathrm{brs}, \mathrm{NH}_{2}\right), 5.80(1 \mathrm{H}, \mathrm{s}$, isoxazole $), 6.46$ $(1 \mathrm{H}, \mathrm{d}, J=8.0, \mathrm{H} 6), 7.26(1 \mathrm{H}, \mathrm{d}, J=8.0, \mathrm{H} 5), 7.27(1 \mathrm{H}, \mathrm{s}, \mathrm{H} 3) \mathrm{ppm}$.

$N^{\prime}$-hydroxy-3-methyl-4-(4-((3-methylisoxazol-5-yl)methyl)piperazin-1-yl)benzimidamide 27b, White solid, yield $47 \%$, m.p. $169-170{ }^{\circ} \mathrm{C}(\mathrm{EtOH})$. MS (EI), $\mathrm{m} / z\left(\mathrm{I}_{\text {relat }} .(\%)\right): 329[\mathrm{M}]^{+}(54)$. Calc. $329.3968, \mathrm{C}_{17} \mathrm{H}_{23} \mathrm{~N}_{5} \mathrm{O}_{2}$. ${ }^{1} \mathrm{H}-\mathrm{NMR}$ (DMSO- $\left.d_{6}\right): \delta 2.22\left(3 \mathrm{H}, \mathrm{s}, \mathrm{CH}_{3}\right), 2.30\left(3 \mathrm{H}, \mathrm{s}, \mathrm{CH}_{3} \mathrm{Ph}\right), 2.73\left(4 \mathrm{H}, \mathrm{m}, \mathrm{N}\left(\mathrm{CH}_{2}\right)_{2}\right), 3.07(4 \mathrm{H}, \mathrm{brt}$, $\left.\mathrm{N}\left(\mathrm{CH}_{2}\right)_{2}\right), 4.12\left(2 \mathrm{H}\right.$, brs, $\left.\mathrm{NCH}_{2}\right), 4.96(1 \mathrm{H}, \mathrm{s}, \mathrm{NOH}), 5.01\left(2 \mathrm{H}, \mathrm{brs}, \mathrm{NH}_{2}\right), 6.30(1 \mathrm{H}, \mathrm{s}$, isoxazole $), 6.46$ $(1 \mathrm{H}, \mathrm{d}, J=9.0, \mathrm{H} 6), 7.26(1 \mathrm{H}, \mathrm{d}, J=9.0, \mathrm{H} 5), 7.27(1 \mathrm{H}, \mathrm{s}, \mathrm{H} 3) \mathrm{ppm}$.

4-(4-Acetylpiperazin-1-yl)-N'-hydroxy-3-methylbenzimidamide 32, White solid, yield 50\%, m.p. 230-232 ${ }^{\circ} \mathrm{C}$ (MeOH). MS (EI), $m / z\left(I_{\text {relat. }}(\%)\right): 276[\mathrm{M}]^{+}(61)$. Calc. $276.3342, \mathrm{C}_{14} \mathrm{H}_{20} \mathrm{~N}_{4} \mathrm{O}_{2} .{ }^{1} \mathrm{H}-\mathrm{NMR}$ (DMSO- $\left.d_{6}\right): \delta$ $1.93\left(3 \mathrm{H}, \mathrm{s}, \mathrm{CH}_{3}\right), 2.22\left(3 \mathrm{H}, \mathrm{s}, \mathrm{CH}_{3} \mathrm{Ph}\right), 3.29\left(4 \mathrm{H}\right.$, brs, $\left.\mathrm{N}\left(\mathrm{CH}_{2}\right)_{2}\right), 3.63\left(4 \mathrm{H}\right.$, brs, $\left.\mathrm{N}\left(\mathrm{CH}_{2}\right)_{2}\right), 4.96(1 \mathrm{H}, \mathrm{s}$, $\mathrm{NOH}), 5.03\left(2 \mathrm{H}, \mathrm{brs}, \mathrm{NH}_{2}\right), 6.46(1 \mathrm{H}, \mathrm{d}, J=7.9, \mathrm{H} 6), 7.26(1 \mathrm{H}, \mathrm{d}, J=7.9, \mathrm{H} 5), 7.27(1 \mathrm{H}, \mathrm{s}, \mathrm{H} 3) \mathrm{ppm}$.

\subsubsection{General Procedure for the Synthesis of Compounds 4, 10, 17, 28a, b, 33}

To a solution of $\mathbf{3}, \mathbf{9}, \mathbf{1 6}, \mathbf{2 7} \mathbf{a}, \mathbf{b}$, or $32(1 \mathrm{mmol})$ in of pyridine heated to $80-90{ }^{\circ} \mathrm{C}$ carefully add dropwise trifluoroacetic anhydride $(2 \mathrm{mmol})$ during $30 \mathrm{~min}$. The reaction mixture was stored for $1 \mathrm{~h}$ at $85^{\circ} \mathrm{C}$. The cooled to rt mixture was diluted with water and extracted with ethyl acetate ( 3 times). The combined organic phases were washed with water ( 3 times), dried over anhydrous $\mathrm{Na}_{2} \mathrm{SO}_{4}$, and concentrated in vacuo. The residue was treated by water and stored in the refrigerator for $2-4 \mathrm{~h}$. Crystals were collected and recrystallized from the corresponding solvent (in parentheses following mp data).

3-(3-Methyl-4-(pent-4-yn-1-ylthio)phenyl)-5-(trifluoromethyl)-1,2,4-oxadiazole 4, White solid, yield 54\%, $\mathrm{mp} 49-50{ }^{\circ} \mathrm{C}(\mathrm{iPrOH})$. MS (EI), $m / z\left(\mathrm{I}_{\text {relat. }}(\%)\right): 326[\mathrm{M}]^{+}(76)$. Calc. $326.3367, \mathrm{C}_{15} \mathrm{H}_{13} \mathrm{~F}_{3} \mathrm{~N}_{2} \mathrm{OS} .{ }^{1} \mathrm{H}-\mathrm{NMR}$ (DMSO- $\left.d_{6}\right): \delta 1.86\left(2 \mathrm{H}\right.$, quint, $\left.J=7.2, \mathrm{CH}_{2} \mathrm{CH}_{2} \mathrm{CH}_{2} \mathrm{~S}\right), 2.21\left(3 \mathrm{H}, \mathrm{s}, \mathrm{CH}_{3} \mathrm{Ph}\right), 2.37(2 \mathrm{H}, \mathrm{t}, J=7.2$, $\left.\mathrm{CH}_{2} \mathrm{CH}_{2} \mathrm{CH}_{2} \mathrm{~S}\right), 2.77\left(1 \mathrm{H}, \mathrm{s}, \mathrm{CHCCH}_{2}\right), 3.25\left(2 \mathrm{H}, \mathrm{t}, J=7.2, \mathrm{CH}_{2} \mathrm{CH}_{2} \mathrm{CH}_{2} \mathrm{~S}\right), 7.47(1 \mathrm{H}, \mathrm{d}, J=7.5, \mathrm{H} 6), 7.59$ $(1 \mathrm{H}, \mathrm{d}, J=7.5, \mathrm{H} 5), 7.64(1 \mathrm{H}, \mathrm{s}, \mathrm{H} 3) \mathrm{ppm}$.

2-Methyl-4-(5-(trifluoromethyl)-1,2,4-oxadiazol-3-yl)phenol 10, White solid, yield 43\%, m.p. $64-65{ }^{\circ} \mathrm{C}$ (Hexane). MS (EI), $m / z\left(I_{\text {relat. }}(\%)\right): 244[\mathrm{M}]^{+}(64)$. Calc. $244.1700, \mathrm{C}_{10} \mathrm{H}_{7} \mathrm{~F}_{3} \mathrm{~N}_{2} \mathrm{O}_{2} \cdot{ }^{1} \mathrm{H}-\mathrm{NMR}\left(\mathrm{DMSO}-d_{6}\right)$ : $\delta 2.19\left(3 \mathrm{H}, \mathrm{s}, \mathrm{CH}_{3} \mathrm{Ph}\right), 7.05(1 \mathrm{H}, \mathrm{d}, J=7.5, \mathrm{H} 6), 7.62(1 \mathrm{H}, \mathrm{d}, J=7.5, \mathrm{H} 5), 7.99(1 \mathrm{H}, \mathrm{s}, \mathrm{H} 3) \mathrm{ppm}$.

Tert-butyl (2-methyl-4-(5-(trifluoromethyl)-1,2,4-oxadiazol-3-yl)phenyl)carbamate 17, White solid, yield 48\%, m.p. $104-106{ }^{\circ} \mathrm{C}(\mathrm{iPrOH})$. MS (EI), $m / z\left(I_{\text {relat. }}(\%)\right): 343[\mathrm{M}]^{+}(58)$. Calc. $343.3010, \mathrm{C}_{15} \mathrm{H}_{16} \mathrm{~F}_{3} \mathrm{~N}_{3} \mathrm{O}_{3}$. ${ }^{1} \mathrm{H}-\mathrm{NMR}\left(\mathrm{DMSO}-d_{6}\right): \delta 1.43(9 \mathrm{H}, \mathrm{s}, \mathrm{tBu}), 2.16\left(3 \mathrm{H}, \mathrm{s}, \mathrm{CH}_{3} \mathrm{Ph}\right), 7.53(1 \mathrm{H}, \mathrm{d}, J=7.5, \mathrm{H} 6), 7.62(1 \mathrm{H}, \mathrm{d}$, $J=7.5, \mathrm{H} 5), 7.65(1 \mathrm{H}, \mathrm{s}, \mathrm{H} 3) \mathrm{ppm}$.

N,N-Dimethyl-5-(4-(2-methyl-4-(5-(trifluoromethyl)-1,2,4-oxadiazol-3-yl)phenyl)piperazin-1-yl)iso-xazole-3carboxamide 28a, White solid, yield 59\%, m.p. $171-173{ }^{\circ} \mathrm{C}(\mathrm{EtOH}) . \mathrm{MS}(\mathrm{EI}), \mathrm{m} / \mathrm{z}\left(\mathrm{I}_{\text {relat. }}(\%)\right): 450[\mathrm{M}]^{+}$ (53). Calc. 450.4143, $\mathrm{C}_{20} \mathrm{H}_{21} \mathrm{~F}_{3} \mathrm{~N}_{6} \mathrm{O}_{3} .{ }^{1} \mathrm{H}$ NMR (DMSO-d 6 ): $\delta 2.39\left(3 \mathrm{H}, \mathrm{s}, \mathrm{CH}_{3} \mathrm{Ph}\right), 2.98\left(3 \mathrm{H}, \mathrm{s}, \mathrm{NCH}_{3}\right)$, 3.04-3.11 (7H, m, $\left.\mathrm{N}\left(\mathrm{CH}_{2}\right)_{2}, \mathrm{NCH}_{3}\right), 3.52\left(4 \mathrm{H}, \mathrm{brt}, \mathrm{N}\left(\mathrm{CH}_{2}\right)_{2}\right), 5.54(1 \mathrm{H}, \mathrm{s}$, isoxazole), $7.23(1 \mathrm{H}, \mathrm{d}, J=8.0$, 
H6), $7.86(1 \mathrm{H}, \mathrm{d}, J=8.0, \mathrm{H} 5), 7.87(1 \mathrm{H}, \mathrm{s}, \mathrm{H} 3) \mathrm{ppm} .{ }^{13} \mathrm{C}-\mathrm{NMR}\left(\mathrm{DMSO}-\mathrm{d}_{6}\right): \delta 17.88,35.15,35.15,46.54$, $46.54,49.96,49.96,86.16,115.89$ (q, $J=273.4), 118.10,120.00,126.97,128.15,129.03,149.89,156.44$, $163.02,166.00,167.12(\mathrm{q}, J=43.0), 171.16 \mathrm{ppm}$.

3-(3-Methyl-4-(4-((3-methylisoxazol-5-yl)methyl)piperazin-1-yl)phenyl)-5-(trifluoromethyl)-1,2,4-oxadiazole 28b, White solid, yield 31\%, mp $94-96{ }^{\circ} \mathrm{C}\left(\mathrm{CCl}_{4}\right)$. MS (EI), $m / z\left(I_{\text {relat. }}(\%)\right): 407[\mathrm{M}]^{+}(48)$. Calc. 407.3896, $\mathrm{C}_{19} \mathrm{H}_{20} \mathrm{~F}_{3} \mathrm{~N}_{5} \mathrm{O}_{2} .{ }^{1} \mathrm{H}-\mathrm{NMR}$ (DMSO- $\left.d_{6}\right): \delta 2.28\left(3 \mathrm{H}, \mathrm{s}, \mathrm{CH}_{3}\right), 2.34\left(3 \mathrm{H}, \mathrm{s}, \mathrm{CH}_{3} \mathrm{Ph}\right), 3.14\left(4 \mathrm{H}, \mathrm{m}, \mathrm{N}_{(}\left(\mathrm{CH}_{2}\right)_{2}\right)$, $3.52\left(4 \mathrm{H}\right.$, brt, $\left.\mathrm{N}\left(\mathrm{CH}_{2}\right)_{2}\right), 4.37\left(2 \mathrm{H}, \mathrm{brs}, \mathrm{NCH}_{2}\right), 6.55(1 \mathrm{H}, \mathrm{s}$, isoxazole $), 7.21(1 \mathrm{H}, \mathrm{d}, J=9.0, \mathrm{H} 6), 7.86(1 \mathrm{H}$, d, $J=9.0, \mathrm{H} 5), 7.87(1 \mathrm{H}, \mathrm{s}, \mathrm{H} 3) \mathrm{ppm} .{ }^{13} \mathrm{C}-\mathrm{NMR}\left(\mathrm{DMSO}-d_{6}\right): \delta 11.26,17.81,50.00,50.00,52.11,52.79$, $52.79,103.88,115.23(q, J=273.1), 118.17,120.00,127.61,128.68,129.80,149.91,159.00,165.99,166.00$, $167.15(\mathrm{q}, J=43.5) \mathrm{ppm}$.

1-(4-(2-methyl-4-(5-(trifluoromethyl)-1,2,4-oxadiazol-3-yl)phenyl)piperazin-1-yl)ethanone 33, White solid, yield $62 \%$, m.p. $65-67^{\circ} \mathrm{C}$ (Hexane). MS (EI), $m / z\left(I_{\text {relat. }}(\%)\right): 354[\mathrm{M}]^{+}(59)$. Calc. $354.3270, \mathrm{C}_{16} \mathrm{H}_{17} \mathrm{~F}_{3} \mathrm{~N}_{4} \mathrm{O}_{2}$. ${ }^{1} \mathrm{H}-\mathrm{NMR}$ (DMSO- $\left.d_{6}\right): \delta 1.93\left(3 \mathrm{H}, \mathrm{s}, \mathrm{CH}_{3}\right), 2.21\left(3 \mathrm{H}, \mathrm{s}, \mathrm{CH}_{3} \mathrm{Ph}\right), 3.30\left(4 \mathrm{H}, \mathrm{brs}, \mathrm{N}\left(\mathrm{CH}_{2}\right)_{2}\right), 3.63(4 \mathrm{H}, \mathrm{brs}$, $\left.\mathrm{N}\left(\mathrm{CH}_{2}\right)_{2}\right), 7.00(1 \mathrm{H}, \mathrm{d}, J=7.9, \mathrm{H} 6), 7.43(1 \mathrm{H}, \mathrm{d}, J=7.9, \mathrm{H} 5), 7.56(1 \mathrm{H}, \mathrm{s}, \mathrm{H} 3) \mathrm{ppm}$.

\subsubsection{General Procedure for the Synthesis of Compounds 6a, 12a, 20a, 28d}

To a solution of 5 or $\mathbf{3 6}$ ( $3 \mathrm{mmol}$ ) in DMF, a solution of corresponding compounds 4 , or $\mathbf{1 1}$, or $\mathbf{1 9}$, or 37 in DMF for 20-30 min was added and stirred at $\mathrm{rt}$ for 40-50 min. To the reaction solution, $\mathrm{Et}_{3} \mathrm{~N}$ ( $3 \mathrm{mmol}$ ) in DMF was added at $80-90{ }^{\circ} \mathrm{C}$ for $2 \mathrm{~h}$. The mixture was stirred at $80-90{ }^{\circ} \mathrm{C}$ for $1-2 \mathrm{~h}$ and at $\mathrm{rt}$ for $12 \mathrm{~h}$. The reaction mixture was diluted with water and extracted with ethyl acetate (3-times). The combined organic phases were washed with water (3-times), dried over anhydrous $\mathrm{Na}_{2} \mathrm{SO}_{4}$, and concentrated in vacuo. The residue was treated by water and stored in the refrigerator for $2-4 \mathrm{~h}$. Crystals were collected and recrystallized from the corresponding solvent (in parentheses following mp data).

3-(3-Methyl-4-((3-(3-carbethoxy-isoxazol-5-yl)propyl)thio)phenyl)-5-(trifluoromethyl)-1,2,4-oxadia-zole 6a, White solid, yield $40 \%$, m.p. $97-100{ }^{\circ} \mathrm{C}(\mathrm{MeOH})$. MS (EI), $m / z\left(I_{\text {relat. }}(\%)\right): 441[\mathrm{M}]^{+}$(65). Calc. 441.4241, $\mathrm{C}_{19} \mathrm{H}_{18} \mathrm{~F}_{3} \mathrm{~N}_{3} \mathrm{O}_{4} \mathrm{~S} .{ }^{1} \mathrm{H}-\mathrm{NMR}$ (DMSO- $\left.d_{6}\right): \delta 1.27\left(3 \mathrm{H}, \mathrm{t}, J=7.1, \mathrm{CH}_{3} \mathrm{CH}_{2} \mathrm{O}\right), 2.05$ (2H, quint, $J=7.5$, $\left.J=7.4, \mathrm{CH}_{2} \mathrm{CH}_{2} \mathrm{CH}_{2} \mathrm{~S}\right), 2.31\left(3 \mathrm{H}, \mathrm{s}, \mathrm{CH}_{3} \mathrm{Ph}\right), 3.01\left(2 \mathrm{H}, \mathrm{t}, J=7.5, \mathrm{CH}_{2} \mathrm{CH}_{2} \mathrm{CH}_{2} \mathrm{~S}\right), 3.11(2 \mathrm{H}, \mathrm{t}, J=7.5$, $\left.\mathrm{CH}_{2} \mathrm{CH}_{2} \mathrm{CH}_{2} \mathrm{~S}\right), 4.32\left(2 \mathrm{H}, \mathrm{q}, J=7.1, \mathrm{CH}_{3} \mathrm{CH}_{2} \mathrm{O}\right), 6.74(1 \mathrm{H}, \mathrm{s}$, isoxazole), $7.47(1 \mathrm{H}, \mathrm{d}, J=8.5, \mathrm{H} 6), 7.84$ $(2 \mathrm{H}, \mathrm{d}, J=8.5, \mathrm{H} 3, \mathrm{H} 5)$ ppm. ${ }^{13} \mathrm{C}-\mathrm{NMR}\left(\mathrm{DMSO}-d_{6}\right): \delta 13.87,19.49,25.03,25.97,29.79,61.67,101.95$, $115.76(\mathrm{q}, J=273.7), 120.39,125.38,125.61,128.15,136.27,141.90,156.09,159.50,164.60(\mathrm{q}, J=43.0)$, $168.24,174.67 \mathrm{ppm}$.

Ethyl 5-(3-(2-methyl-4-(5-(trifluoromethyl)-1,2,4-oxadiazol-3-yl)phenoxy)-3-oxopropyl)-isoxazole-3-carboxylate 12a, White solid, yield $34 \%$, m.p. $95-96{ }^{\circ} \mathrm{C}$ (Hexane). MS (EI), $m / z\left(I_{\text {relat. }}(\%)\right): 439[\mathrm{M}]^{+}(41)$. Calc. 439.3420, $\mathrm{C}_{19} \mathrm{H}_{16} \mathrm{~F}_{3} \mathrm{~N}_{3} \mathrm{O}_{6} .{ }^{1} \mathrm{H}-\mathrm{NMR}$ (DMSO- $\left.d_{6}\right): \delta 1.32\left(3 \mathrm{H}, \mathrm{t}, J=7.1, \mathrm{CH}_{3} \mathrm{CH}_{2} \mathrm{O}\right), 2.20(3 \mathrm{H}, \mathrm{s}$, $\left.\mathrm{CH}_{3} \mathrm{Ph}\right), 3.20-3.27\left(4 \mathrm{H}, \mathrm{m}, \mathrm{CH}_{2} \mathrm{CH}_{2} \mathrm{CO}\right), 4.37\left(2 \mathrm{H}, \mathrm{q}, J=7.1, \mathrm{CH}_{3} \mathrm{CH}_{2} \mathrm{O}\right), 6.79(1 \mathrm{H}, \mathrm{s}$, isoxazole), 7.33 $(1 \mathrm{H}, \mathrm{d}, J=8.4, \mathrm{H} 6), 7.94(1 \mathrm{H}, \mathrm{d}, J=8.4, \mathrm{H} 5), 8.01(1 \mathrm{H}, \mathrm{s}, \mathrm{H} 3) \mathrm{ppm} .{ }^{13} \mathrm{C}-\mathrm{NMR}$ (DMSO- $\left.d_{6}\right): \delta 13.64,15.52$, $21.43,31.14,60.95,102.05,115.84(\mathrm{q}, J=273.6), 121.94,122.20,123.45,126.23,130.13,131.78,152.16$, $159.88,166.10(\mathrm{q}, J=42.8), 167.84,169.66,171.15 \mathrm{ppm}$.

Ethyl 5-(3-((2-methyl-4-(5-(trifluoromethyl)-1,2,4-oxadiazol-3-yl)phenyl)amino)-3-oxopropyl)isoxa-zole-3carboxylate 20a, White solid, yield 78\%, m.p. $166-168^{\circ} \mathrm{C}(\mathrm{EtOH}) . \mathrm{MS}(\mathrm{EI}), \mathrm{m} / z\left(\mathrm{I}_{\text {relat }} .(\%)\right): 438[\mathrm{M}]^{+}$ (56). Calc. 438.3573, $\mathrm{C}_{19} \mathrm{H}_{17} \mathrm{~F}_{3} \mathrm{~N}_{4} \mathrm{O}_{5} .{ }^{1} \mathrm{H}-\mathrm{NMR}$ (DMSO- $\left.d_{6}\right): \delta 1.31\left(3 \mathrm{H}, \mathrm{t}, J=7.1, \mathrm{CH}_{3} \mathrm{CH}_{2} \mathrm{O}\right), 2.31(3 \mathrm{H}$, $\left.\mathrm{s}, \mathrm{CH}_{3} \mathrm{Ph}\right), 2.89\left(2 \mathrm{H}, \mathrm{t}, J=7.1, \mathrm{CH}_{2} \mathrm{CH}_{2} \mathrm{CO}\right), 3.17\left(2 \mathrm{H}, \mathrm{t}, J=7.1, \mathrm{CH}_{2} \mathrm{CH}_{2} \mathrm{CO}\right), 4.36(2 \mathrm{H}, \mathrm{q}, J=7.1$, $\left.\mathrm{CH}_{3} \mathrm{CH}_{2} \mathrm{O}\right), 6.70(1 \mathrm{H}, \mathrm{s}$, isoxazole), $7.79(1 \mathrm{H}, \mathrm{d}, J=8.8, \mathrm{H} 6), 7.87(1 \mathrm{H}, \mathrm{dd}, J=1.8,8.8, \mathrm{H} 5), 7.91(1 \mathrm{H}, \mathrm{s}$, H3), $9.53(1 \mathrm{H}, \mathrm{brs}, \mathrm{NH}) \mathrm{ppm} .{ }^{13} \mathrm{C}-\mathrm{NMR}$ (DMSO- $\left.d_{6}\right): \delta 13.88,17.69,21.97,32.91,61.70,101.76,115.73(\mathrm{q}$, $J=273.4), 120.31,124.54,125.31,129.29,131.62,140.21,156.05,159.50,164.84(\mathrm{q}, J=43.9), 168.14,169.66$, $174.86 \mathrm{ppm}$. 
Methyl 5-(4-(2-methyl-4-(5-(trifluoromethyl)-1,2,4-oxadiazol-3-yl)phenyl)piperazin-1-yl)-isoxazole-3-carboxylate 28d, White solid, yield 53\%, m.p. $113-115{ }^{\circ} \mathrm{C}(\mathrm{EtOH}) . \mathrm{MS}(\mathrm{EI}), \mathrm{m} / z\left(I_{\text {relat }}(\%)\right)$ : $451[\mathrm{M}]^{+}(72)$. Calc. 451.3991, $\mathrm{C}_{20} \mathrm{H}_{20} \mathrm{~F}_{3} \mathrm{~N}_{5} \mathrm{O}_{4} .{ }^{1} \mathrm{H}-\mathrm{NMR}$ (DMSO- $\left.d_{6}\right): \delta 2.31\left(3 \mathrm{H}, \mathrm{s}, \mathrm{CH}_{3} \mathrm{Ph}\right), 2.64\left(4 \mathrm{H}, \mathrm{brs}, \mathrm{N}\left(\mathrm{CH}_{2}\right)_{2}\right)$, $2.97\left(4 \mathrm{H}\right.$, brs, $\left.\mathrm{N}\left(\mathrm{CH}_{2}\right)_{2}\right), 3.87\left(2 \mathrm{H}, \mathrm{s}, \mathrm{PhCH}_{2} \mathrm{~N}\right), 3.90\left(3 \mathrm{H}, \mathrm{s}, \mathrm{CH}_{3} \mathrm{O}\right), 6.87(1 \mathrm{H}, \mathrm{s}$, isoxazole $), 7.17(1 \mathrm{H}$, $\mathrm{d}, J=8.4, \mathrm{H} 6), 7.83(1 \mathrm{H}, \mathrm{d}, J=8.4, \mathrm{H} 5), 7.84(1 \mathrm{H}, \mathrm{s}, \mathrm{H} 3) \mathrm{ppm} .{ }^{13} \mathrm{C}-\mathrm{NMR}\left(\mathrm{DMSO}-d_{6}\right): \delta 17.89,49.87$, $49.87,52.15,52.81,52.81,53.11,104.05,115.34$ (q, $J=273.2), 118.14,120.03,127.67,128.57,129.85,150.23$, $159.12,160.31,166.05,166.14,167.32(\mathrm{q}, J=43.3) \mathrm{ppm}$.

\subsubsection{General Procedure for the Synthesis of Compounds $\mathbf{6 b}, \mathbf{1 2 b}, \mathbf{2 0 b}$}

To a solution of NCS (2.5 mmol) and 1-2 drops of pyridine in DMF, a solution of acetaldoxime $(2.5 \mathrm{mmol})$ in DMF was added for $30 \mathrm{~min}$ and stirred at $\mathrm{rt}$ for $1 \mathrm{~h}$; then, to the solution, a solution of 4 , or 11, or 19 ( $1 \mathrm{mmol})$ in DMF was added for $20 \mathrm{~min}$. To the resulted mixture, $\mathrm{Et}_{3} \mathrm{~N}(2.5 \mathrm{mmol})$ in DMF was added at $80-90^{\circ} \mathrm{C}$ for $1 \mathrm{~h}$ and stirred at $80-90^{\circ} \mathrm{C}$ for $3-4 \mathrm{~h}$. The reaction mixture was diluted with water and extracted with ethyl acetate (3 times). The combined organic phases were washed with water ( 3 times), dried over anhydrous $\mathrm{Na}_{2} \mathrm{SO}_{4}$, and concentrated in vacuo. The residue was recrystallized from the corresponding solvent (in parentheses following mp data).

3-(3-Methyl-4-((3-(3-methylisoxazol-5-yl)propyl)thio)phenyl)-5-(trifluoromethyl)-1,2,4-oxadiazole 6b, White solid, yield 32\%, m.p. $60-63{ }^{\circ} \mathrm{C}$ (Hexane). MS (EI), $m / z\left(I_{\text {relat. }}(\%)\right): 383[\mathrm{M}]^{+}(41)$. Calc. 383.3880, $\mathrm{C}_{17} \mathrm{H}_{16} \mathrm{~F}_{3} \mathrm{~N}_{3} \mathrm{O}_{2} \mathrm{~S} .{ }^{1} \mathrm{H}-\mathrm{NMR}$ (DMSO-d $): \delta 2.00$ (2H, quint, $J=7.2, \mathrm{CH}_{2} \mathrm{CH}_{2} \mathrm{CH}_{2} \mathrm{~S}$ ), $2.18\left(3 \mathrm{H}, \mathrm{s}, \mathrm{CH}_{3}\right)$, $2.35\left(3 \mathrm{H}, \mathrm{s}, \mathrm{CH}_{3} \mathrm{Ph}\right), 2.89\left(2 \mathrm{H}, \mathrm{t}, J=7.2, \mathrm{CH}_{2} \mathrm{CH}_{2} \mathrm{CH}_{2} \mathrm{~S}\right), 3.13\left(2 \mathrm{H}, \mathrm{t}, J=7.2, \mathrm{CH}_{2} \mathrm{CH}_{2} \mathrm{CH}_{2} \mathrm{~S}\right), 6.14(1 \mathrm{H}, \mathrm{s}$, isoxazole), $7.48(1 \mathrm{H}, \mathrm{d}, J=7.5, \mathrm{H} 6), 7.85(1 \mathrm{H}, \mathrm{d}, J=7.5, \mathrm{H} 5), 7.87(1 \mathrm{H}, \mathrm{s}, \mathrm{H} 3) \mathrm{ppm} .{ }^{13} \mathrm{C}-\mathrm{NMR}\left(\mathrm{DMSO}-d_{6}\right)$ : $\delta 11.05,19.53,24.96,26.04,29.84,101.76,115.78$ (q, $J=273.1), 120.38,125.40,125.62,128.19,136.25$, $141.92,159.95,164.71(\mathrm{q}, J=43.4), 168.25,170.65 \mathrm{ppm}$.

2-Methyl-4-(5-(trifluoromethyl)-1,2,4-oxadiazol-3-yl)phenyl 3-(3-methylisoxazol-5-yl)propanoate 12b, White solid, yield 29\%, mp 67-69 ${ }^{\circ} \mathrm{C}$ (Hexane). MS (EI), m/z (I relat. $\%$ )): $381[\mathrm{M}]^{+}$(53). Calc. 381.3060, $\mathrm{C}_{17} \mathrm{H}_{14} \mathrm{~F}_{3} \mathrm{~N}_{3} \mathrm{O}_{4} .{ }^{1} \mathrm{H}-\mathrm{NMR}$ (DMSO-d $)$ : $\delta 2.18\left(3 \mathrm{H}, \mathrm{s}, \mathrm{CH}_{3}\right), 2.35\left(3 \mathrm{H}, \mathrm{s}, \mathrm{CH}_{3} \mathrm{Ph}\right), 3.20-3.27(4 \mathrm{H}, \mathrm{m}$, $\left.\mathrm{CH}_{2} \mathrm{CH}_{2} \mathrm{CO}\right), 6.14(1 \mathrm{H}, \mathrm{s}$, isoxazole), $7.48(1 \mathrm{H}, \mathrm{d}, J=7.5, \mathrm{H} 6), 7.85(1 \mathrm{H}, \mathrm{d}, J=7.5, \mathrm{H} 5), 7.87(1 \mathrm{H}, \mathrm{s}, \mathrm{H} 3)$ ppm. ${ }^{13}$ C-NMR (DMSO- $\left.d_{6}\right): \delta 10.89,15.48,21.45,31.06,102.16,115.77(q, J=273.4), 122.15,123.44$, $126.34,130.05,131.67,152.12,159.46,165.01$ (q, $J=42.8), 167.92,169.86,170.97$ ppm.

N-(2-Methyl-4-(5-(trifluoromethyl)-1,2,4-oxadiazol-3-yl)phenyl)-3-(3-methylisoxazol-5-yl)propan-amide 20b, White solid, yield 83\%, m.p. 176-179 ${ }^{\circ} \mathrm{C}(\mathrm{EtOH})$. MS (EI), $m / z\left(I_{\text {relat. }}(\%)\right): 380[\mathrm{M}]^{+}(68)$. Calc. 380.3212, $\mathrm{C}_{17} \mathrm{H}_{15} \mathrm{~F}_{3} \mathrm{~N}_{4} \mathrm{O}_{3} .{ }^{1} \mathrm{H}-\mathrm{NMR}\left(\mathrm{DMSO}-d_{6}\right): \delta 2.19\left(3 \mathrm{H}, \mathrm{s}, \mathrm{CH}_{3}\right), 2.31\left(3 \mathrm{H}, \mathrm{s}, \mathrm{CH}_{3} \mathrm{Ph}\right), 2.82(2 \mathrm{H}, \mathrm{t}, \mathrm{J}=7.3$, $\left.\mathrm{CH}_{2} \mathrm{CH}_{2} \mathrm{CO}\right), 3.05\left(2 \mathrm{H}, \mathrm{t}, J=7.3, \mathrm{CH}_{2} \mathrm{CH}_{2} \mathrm{CO}\right), 6.13(1 \mathrm{H}, \mathrm{s}$, isoxazole $), 7.81(1 \mathrm{H}, \mathrm{d}, J=8.5, \mathrm{H} 6), 7.87(1 \mathrm{H}$, $\mathrm{dd}, J=1.8,8.5, \mathrm{H} 5), 7.91(1 \mathrm{H}, \mathrm{s}, \mathrm{H} 3), 9.49(1 \mathrm{H}, \mathrm{brs}, \mathrm{NH}) \mathrm{ppm} .{ }^{13} \mathrm{C}-\mathrm{NMR}\left(\mathrm{DMSO}-d_{6}\right): \delta 11.15,17.68$, $21.87,33.12,100.97,115.75(q, J=273.1), 120.33,124.45,125.30,129.27,121.67,140.15,159.86,164.80(q$, $J=43.5), 168.10,169.69,173.97$ ppm.

\subsubsection{General Procedure for the Synthesis of Compounds 7, 21, 26}

A mixture of $\mathbf{6 a}$, or $\mathbf{2 0 a}$, or $\mathbf{2 5 a}$ and dimethylamine solution $17 \mathrm{wt}$ \% in dioxane was heated at 50-60 ${ }^{\circ} \mathrm{C}$ for $1-12 \mathrm{~h}$. The cooled reaction mixture was concentrated in vacuo. The residue was treated by water and stored in the refrigerator for $12 \mathrm{~h}$. Crystals were collected and recrystallized from the corresponding solvent (in parentheses following mp data).

N,N-dimethyl-5-(3-((2-methyl-4-(5-(trifluoromethyl)-1,2,4-oxadiazol-3-yl)phenyl)thio)propyl)isoxa-zole-3carboxamide 7, White solid, yield 57\%, m.p. 95.5-97 ${ }^{\circ} \mathrm{C}$ (Hexane). MS (EI), m/z (I relat. $\left.(\%)\right): 440[\mathrm{M}]^{+}(84)$. Calc. 440.4394, $\mathrm{C}_{19} \mathrm{H}_{19} \mathrm{~F}_{3} \mathrm{~N}_{4} \mathrm{O}_{3} \mathrm{~S}$. ${ }^{1} \mathrm{H}-\mathrm{NMR}$ (DMSO-d 6 ): $\delta 2.05$ (2H, quint, $J=7.2, \mathrm{CH}_{2} \mathrm{CH}_{2} \mathrm{CH}_{2} \mathrm{~S}$ ), 2.34 $\left(3 \mathrm{H}, \mathrm{s}, \mathrm{CH}_{3} \mathrm{Ph}\right), 2.99\left(3 \mathrm{H}, \mathrm{s}, \mathrm{NCH}_{3}\right), 3.05\left(3 \mathrm{H}, \mathrm{s}, \mathrm{NCH}_{3}\right), 3.02\left(2 \mathrm{H}, \mathrm{t}, J=7.2, \mathrm{CH}_{2} \mathrm{CH}_{2} \mathrm{CH}_{2} \mathrm{~S}\right), 3.15(2 \mathrm{H}, \mathrm{t}$, $\left.J=7.2, \mathrm{CH}_{2} \mathrm{CH}_{2} \mathrm{CH}_{2} \mathrm{~S}\right), 6.52(1 \mathrm{H}, \mathrm{s}$, isoxazole $), 7.47(1 \mathrm{H}, \mathrm{s}, J=7.5, \mathrm{H} 6), 7.85(1 \mathrm{H}, \mathrm{d}, J=7.5, \mathrm{H} 5), 7.87(1 \mathrm{H}$, 
s, H3) ppm. ${ }^{13}$ C-NMR (DMSO- $\left.d_{6}\right): \delta 19.50,24.94,26.07,29.86,34.85,37.95,101.66,115.77(\mathrm{q}, J=273.3)$, 120.40, 125.40, 125.59, 128.17, 136.28, 141.94, 158.70, 160.58, 164.85 (q, $J=43.5), 168.24,172.74$ ppm.

N,N-dimethyl-5-(3-((2-methyl-4-(5-(trifluoromethyl)-1,2,4-oxadiazol-3-yl)phenyl)amino)-3-oxopropyl)isoxazole-3-carboxamide 21, White solid, yield 63\%, m.p. $169-171{ }^{\circ} \mathrm{C}$ (Hexane:EtOAc). MS (EI), $m / z\left(\mathrm{I}_{\text {relat. }}(\%)\right): 437[\mathrm{M}]^{+}$(63). Calc. 437.3725, $\mathrm{C}_{19} \mathrm{H}_{18} \mathrm{~F}_{3} \mathrm{~N}_{5} \mathrm{O}_{4} .{ }^{1} \mathrm{H}-\mathrm{NMR}$ (DMSO-d $\left.\mathrm{d}_{6}\right): \delta 2.31(3 \mathrm{H}, \mathrm{s}$, $\left.\mathrm{CH}_{3} \mathrm{Ph}\right), 2.88\left(2 \mathrm{H}, \mathrm{t}, J=7.2, \mathrm{CH}_{2} \mathrm{CH}_{2} \mathrm{CO}\right), 3.00\left(3 \mathrm{H}, \mathrm{s}, \mathrm{NCH}_{3}\right), 3.11\left(3 \mathrm{H}, \mathrm{s}, \mathrm{NCH}_{3}\right), 3.15(2 \mathrm{H}, \mathrm{t}, J=7.2$, $\left.\mathrm{CH}_{2} \mathrm{CH}_{2} \mathrm{CO}\right), 6.49(1 \mathrm{H}, \mathrm{s}$, isoxazole), $7.80(1 \mathrm{H}, \mathrm{d}, J=8.3, \mathrm{H} 6), 7.87(1 \mathrm{H}, \mathrm{dd}, J=2.0,8.3, \mathrm{H} 5), 7.91(1 \mathrm{H}, \mathrm{s}$, H3), $9.53\left(1 \mathrm{H}\right.$, brs, NH) ppm. ${ }^{13} \mathrm{C}-\mathrm{NMR}\left(\mathrm{DMSO}_{\mathrm{d}}\right.$ ): $\delta 17.66,21.82,32.99,34.82,37.86,101.48,115.72(\mathrm{q}$, $J=273.2), 120.25,124.49,125.25,129.22,131.61,140.17,158.61,160.48,164.79(q, J=43.0), 168.09,169.64$, $172.79 \mathrm{ppm}$.

5-(4-(4-Cyano-2-methylphenyl)piperazin-1-yl)-N,N-dimethylisoxazole-3-carboxamide 26, White solid, yield $77 \%$, m.p. $145-147^{\circ} \mathrm{C}(\mathrm{EtOH})$. MS (EI), $m / z\left(I_{\text {relat }} .(\%)\right): 339[\mathrm{M}]^{+}$(72). Calc. $339.3916, \mathrm{C}_{18} \mathrm{H}_{21} \mathrm{~N}_{5} \mathrm{O}_{2}$. ${ }^{1} \mathrm{H}-\mathrm{NMR}$ (DMSO- $\left.d_{6}\right): \delta 2.29\left(3 \mathrm{H}, \mathrm{s}, \mathrm{CH}_{3} \mathrm{Ph}\right), 2.73\left(6 \mathrm{H}, \mathrm{s}, \mathrm{N}\left(\mathrm{CH}_{3}\right)_{2}\right), 3.20\left(4 \mathrm{H}, \mathrm{brt}, \mathrm{N}\left(\mathrm{CH}_{2}\right)_{2}\right), 3.21(4 \mathrm{H}$, brt, $\left.\mathrm{N}\left(\mathrm{CH}_{2}\right)_{2}\right), 5.80(1 \mathrm{H}, \mathrm{s}$, isoxazole $), 6.60(1 \mathrm{H}, \mathrm{d}, J=8.0, \mathrm{H} 6), 7.49(1 \mathrm{H}, \mathrm{d}, J=8.0, \mathrm{H} 5), 7.64(1 \mathrm{H}, \mathrm{s}$, H3) ppm.

\subsubsection{Synthesis of 2-Methyl-4-(5-(trifluoromethyl)-1,2,4-oxadiazol-3-yl)phenyl pent-4-ynoate 11}

A mixture of $10(1 \mathrm{mmol}), \mathrm{DCC}(2 \mathrm{mmol}), 4$-pentynoic acid $(2 \mathrm{mmol})$ in pyridine was stirred at $\mathrm{rt}$ for $12 \mathrm{~h}$. The mixture was diluted with $\mathrm{CHCl}_{3}$, and precipitated urea was filtered off. The $\mathrm{CHCl}_{3}$ solution was washed with $3 \%$ aq. $\mathrm{HCl}$ and water ( 3 times) and then dried over $\mathrm{Na}_{2} \mathrm{SO}_{4}$. The solution was filtered off through a short silica gel column, and the solvent was concentrated in vacuo. White solid, yield $72 \%$, m.p. $54-56{ }^{\circ} \mathrm{C}$. MS (EI), $m / z\left(I_{\text {relat. }}(\%)\right): 324[\mathrm{M}]^{+}$(78). Calc. $324.2546, \mathrm{C}_{15} \mathrm{H}_{11} \mathrm{~F}_{3} \mathrm{~N}_{2} \mathrm{O}_{3} .{ }^{1} \mathrm{H}-\mathrm{NMR}$ (DMSO- $\left.d_{6}\right): \delta 2.22\left(3 \mathrm{H}, \mathrm{s}, \mathrm{CH}_{3} \mathrm{Ph}\right), 2.51-2.56\left(2 \mathrm{H}\right.$, quint, $\left.\mathrm{CH}_{2} \mathrm{CH}_{2} \mathrm{CO}\right), 2.82\left(2 \mathrm{H}, \mathrm{m}, \mathrm{CH}_{2} \mathrm{CH}_{2} \mathrm{O}\right), 2.86$ $\left(1 \mathrm{H}, \mathrm{s}, \mathrm{CHCCH}_{2}\right), 7.32(1 \mathrm{H}, \mathrm{d}, J=8.4, \mathrm{H} 6), 7.94(1 \mathrm{H}, \mathrm{d}, J=8.4, \mathrm{H} 5), 8.01(1 \mathrm{H}, \mathrm{s}, \mathrm{H} 3) \mathrm{ppm}$.

\subsubsection{Synthesis of tert-Butyl (4-cyano-2-methylphenyl)carbamate 15}

A mixture of $14(1 \mathrm{~mol})$ and $\mathrm{Boc}_{2} \mathrm{O}(3 \mathrm{~mol})$ was refluxed for $48 \mathrm{~h}$. The reaction mixture was diluted with methanol, brought to the boil, and concentrated in vacuo. The procedure was repeated 3 times. The residue was treated by hexane and stored in the refrigerator for 4-6 h. Crystals were collected and recrystallized from ethanol. White solid, yield $93 \%, \mathrm{mp} 89-91{ }^{\circ} \mathrm{C}$. MS (EI), $m / z\left(I_{\text {relat. }}(\%)\right): 232[\mathrm{M}]^{+}(37)$. Calc. 232.2783, $\mathrm{C}_{13} \mathrm{H}_{16} \mathrm{~N}_{2} \mathrm{O}_{2} .{ }^{1} \mathrm{H}-\mathrm{NMR}$ (DMSO- $\left.d_{6}\right): \delta 1.43(9 \mathrm{H}, \mathrm{s}, \mathrm{tBu}), 2.28\left(3 \mathrm{H}, \mathrm{s}, \mathrm{CH}_{3} \mathrm{Ph}\right), 6.88(1 \mathrm{H}, \mathrm{d}$, $J=7.5, \mathrm{H} 6), 7.60(1 \mathrm{H}, \mathrm{d}, J=7.5, \mathrm{H} 5), 7.74(1 \mathrm{H}, \mathrm{s}, \mathrm{H} 3) \mathrm{ppm}$.

\subsubsection{Synthesis of 2-Methyl-4-(5-(trifluoromethyl)-1,2,4-oxadiazol-3-yl)aniline 18}

To a solution of $\mathbf{1 7}(1 \mathrm{mmol})$ in DCM, TFA $(10 \mathrm{mmol})$ was added at $0{ }^{\circ} \mathrm{C}$. The reaction mixture was stirred at $\mathrm{rt}$ for 2-3 h, after which the solvents were removed in vacuo. The residue was triturated with water to provide the product as a solid. Crystals were collected and recrystallized from hexane:EtOAc. White solid, yield $99 \%$, m.p. $118^{\circ} \mathrm{C}$ (decomp.). MS (EI), $m / z\left(I_{\text {relat. }}(\%)\right): 243[\mathrm{M}]^{+}$(81). Calc. 243.1852, $\mathrm{C}_{10} \mathrm{H}_{8} \mathrm{~F}_{3} \mathrm{~N}_{3} \mathrm{O} .{ }^{1} \mathrm{H}-\mathrm{NMR}$ (DMSO- $\left.d_{6}\right): \delta 2.23\left(3 \mathrm{H}, \mathrm{s}, \mathrm{CH}_{3} \mathrm{Ph}\right), 5.27\left(2 \mathrm{H}, \mathrm{brs}, \mathrm{NH}_{2}\right), 6.71(1 \mathrm{H}, \mathrm{d}, J=7.5, \mathrm{H} 6)$, $7.58(1 \mathrm{H}, \mathrm{d}, J=7.5, \mathrm{H} 5), 7.64(1 \mathrm{H}, \mathrm{s}, \mathrm{H} 3) \mathrm{ppm}$.

3.2.10. Synthesis of N-(2-Methyl-4-(5-(trifluoromethyl)-1,2,4-oxadiazol-3-yl)phenyl)pent-4-ynamide 19

To a solution of 18 ( $1 \mathrm{~mol})$ and DMAP (1.3 mol) in DCM, 4-pentynoic acid (1 mol) was added in one portion, followed by the addition of EDCI $(1.3 \mathrm{~mol})$ in one portion at rt. The reaction mixture was stirred at rt overnight, after which it was washed successively with $3 \%$ aq. $\mathrm{HCl}$ and water (3 times). The organic layer was dried over $\mathrm{Na}_{2} \mathrm{SO}_{4}$ and concentrated in vacuo. The residue was recrystallized from ethanol. White solid, yield $38 \%$, m.p. $165-167^{\circ} \mathrm{C}$. MS (EI), $m / z\left(I_{\text {relat. }}(\%)\right): 323[\mathrm{M}]^{+}$ (65). Calc. 323.2699, $\mathrm{C}_{15} \mathrm{H}_{12} \mathrm{~F}_{3} \mathrm{~N}_{3} \mathrm{O}_{2} .{ }^{1} \mathrm{H}-\mathrm{NMR}$ (DMSO- $d_{6}$ ): $\delta 2.16\left(3 \mathrm{H}, \mathrm{s}, \mathrm{CH}_{3} \mathrm{Ph}\right.$ ), 2.50-2.57 (2H, quint, 
$\left.\mathrm{CH}_{2} \mathrm{CH}_{2} \mathrm{CO}\right), 2.62\left(2 \mathrm{H}, \mathrm{m}, \mathrm{CH}_{2} \mathrm{CH}_{2} \mathrm{O}\right), 2.78\left(1 \mathrm{H}, \mathrm{s}, \mathrm{CHCCH}_{2}\right), 7.32(1 \mathrm{H}, \mathrm{d}, J=8.4, \mathrm{H} 6), 7.61(1 \mathrm{H}, \mathrm{d}$, $J=8.4, \mathrm{H} 5), 7.70(1 \mathrm{H}, \mathrm{s}, \mathrm{H} 3), 9.12(1 \mathrm{H}, \mathrm{brs}, \mathrm{NH}) \mathrm{ppm}$.

\subsubsection{General Procedure for the Synthesis of Compounds 25a,b, 28c}

A mixture of the corresponding phenyl-piperazine (1 mol), the corresponding isoxazole $(1.4 \mathrm{~mol})$, and finely divided $\mathrm{K}_{2} \mathrm{CO}_{3}(3 \mathrm{~mol})$ in acetonitrile was refluxed for $24-48 \mathrm{~h}$. The hot mixture was filtered, and the remaining solids were washed with acetonitrile. The combined filtrates were concentrated in vacuo. The residue was recrystallized from ethanol.

Ethyl 5-(4-(4-cyano-2-methylphenyl)piperazin-1-yl)isoxazole-3-carboxylate 25a, Light beige solid, yield $40 \%$, m.p. $124-125{ }^{\circ} \mathrm{C}$. MS (EI), $m / z\left(I_{\text {relat }} .(\%)\right): 340[\mathrm{M}]^{+}(42)$. Calc. 340.3764, $\mathrm{C}_{18} \mathrm{H}_{20} \mathrm{~N}_{4} \mathrm{O}_{3}$. ${ }^{1} \mathrm{H}-\mathrm{NMR}$ (DMSO-d $\left.d_{6}\right): \delta 1.29\left(3 \mathrm{H}, \mathrm{t}, J=7.1, \mathrm{CH}_{3} \mathrm{CH}_{2} \mathrm{O}\right), 2.32\left(3 \mathrm{H}, \mathrm{s}, \mathrm{CH}_{3} \mathrm{Ph}\right), 3.05\left(4 \mathrm{H}, \mathrm{brs}, \mathrm{N}\left(\mathrm{CH}_{2}\right)_{2}\right)$, $3.52\left(4 \mathrm{H}\right.$, brs, $\left.\mathrm{N}\left(\mathrm{CH}_{2}\right)_{2}\right), 4.35\left(2 \mathrm{H}, \mathrm{q}, J=7.1, \mathrm{CH}_{3} \mathrm{CH}_{2} \mathrm{O}\right), 5.75(1 \mathrm{H}, \mathrm{s}$, isoxazole $), 7.19(1 \mathrm{H}, \mathrm{d}, J=7.9, \mathrm{H} 6)$, $7.61(1 \mathrm{H}, \mathrm{d}, J=7.9, \mathrm{H} 5), 7.63(1 \mathrm{H}, \mathrm{s}, \mathrm{H} 3) \mathrm{ppm}$.

3-Methyl-4-(4-((3-methylisoxazol-5-yl)methyl)piperazin-1-yl)benzonitrile 25b, Yellow solid, yield 56\%, m.p. 86-88 ${ }^{\circ} \mathrm{C}$. MS (EI), $m / z\left(I_{\text {relat. }}(\%)\right): 296[\mathrm{M}]^{+}$(57). Calc. 296.3669, $\mathrm{C}_{17} \mathrm{H}_{20} \mathrm{~N}_{4} \mathrm{O} .{ }^{1} \mathrm{H}-\mathrm{NMR}\left(\mathrm{DMSO}-d_{6}\right): \delta$ $2.29\left(3 \mathrm{H}, \mathrm{s}, \mathrm{CH}_{3}\right), 2.30\left(3 \mathrm{H}, \mathrm{s}, \mathrm{CH}_{3} \mathrm{Ph}\right), 2.72\left(4 \mathrm{H}, \mathrm{m}, \mathrm{N}\left(\mathrm{CH}_{2}\right)_{2}\right), 3.02\left(4 \mathrm{H}, \mathrm{m}, \mathrm{N}\left(\mathrm{CH}_{2}\right)_{2}\right), 4.12(2 \mathrm{H}, \mathrm{brs}$, $\left.\mathrm{NCH}_{2}\right), 6.30(1 \mathrm{H}, \mathrm{s}$, isoxazole), $6.60(1 \mathrm{H}, \mathrm{d}, J=9.0, \mathrm{H} 6), 7.49(1 \mathrm{H}, \mathrm{d}, J=9.0, \mathrm{H} 5), 7.64(1 \mathrm{H}, \mathrm{s}, \mathrm{H} 3) \mathrm{ppm}$.

Ethyl 5-(4-(2-methyl-4-(5-(trifluoromethyl)-1,2,4-oxadiazol-3-yl)phenyl)piperazin-1-yl)isoxazole-3-carboxylate 28c, White solid, yield $80 \%$, m.p. $121-123^{\circ} \mathrm{C}$. MS (EI), $\left.m / z\left(I_{\text {relat }} . \%\right)\right): 451[\mathrm{M}]^{+}$(46). Calc. 451.3991 , $\mathrm{C}_{20} \mathrm{H}_{20} \mathrm{~F}_{3} \mathrm{~N}_{5} \mathrm{O}_{4} \cdot{ }^{1} \mathrm{H}-\mathrm{NMR}$ (DMSO-d $\left.d_{6}\right): \delta 1.30\left(3 \mathrm{H}, \mathrm{t}, J=7.1, \mathrm{CH}_{3} \mathrm{CH}_{2} \mathrm{O}\right), 2.38\left(3 \mathrm{H}, \mathrm{s}, \mathrm{CH}_{3} \mathrm{Ph}\right), 3.07(4 \mathrm{H}$, brs, $\left.\mathrm{N}\left(\mathrm{CH}_{2}\right)_{2}\right), 3.54\left(4 \mathrm{H}\right.$, brs, $\left.\mathrm{N}\left(\mathrm{CH}_{2}\right)_{2}\right), 4.32\left(2 \mathrm{H}, \mathrm{q}, J=7.1, \mathrm{CH}_{3} \mathrm{CH}_{2} \mathrm{O}\right), 5.80(1 \mathrm{H}, \mathrm{s}$, isoxazole $), 7.23(1 \mathrm{H}$, $\mathrm{d}, J=7.9, \mathrm{H} 6), 7.86(1 \mathrm{H}, \mathrm{d}, J=7.9, \mathrm{H} 5), 7.87(1 \mathrm{H}, \mathrm{s}, \mathrm{H} 3)$ ppm. ${ }^{13} \mathrm{C}-\mathrm{NMR}$ (DMSO- $\left.d_{6}\right): \delta 13.89,17.82$, $46.58,46.58,49.94,49.94,61.75,87.21,115.73$ (q, $J=273.2), 118.15,120.05,126.94,128.19,128.95,150.07$, $156.42,160.34,162.95,167.10(\mathrm{q}, J=43.1), 171.05 \mathrm{ppm}$.

\subsubsection{Synthesis of 1-(4-(4-Bromo-2-methylphenyl)piperazin-1-yl)ethanone 30}

A mixture of $19(1 \mathrm{~mol})$ and $\mathrm{Ac}_{2} \mathrm{O}(5 \mathrm{~mol})$ was heated at $60-65{ }^{\circ} \mathrm{C}$ for $4-5 \mathrm{~h}$. The reaction mixture was poured into cold water and stirred for $2 \mathrm{~h}$. Precipitate was collected and recrystallized from methanol. White solid, yield 58\%, m.p. $120-121{ }^{\circ} \mathrm{C}$. MS (EI), $m / z\left(I_{\text {relat. }}(\%)\right): 297[\mathrm{M}]^{+}$(73). Calc. 297.1909, $\mathrm{C}_{13} \mathrm{H}_{17} \mathrm{BrN}_{2} \mathrm{O} .{ }^{1} \mathrm{H}-\mathrm{NMR}$ (DMSO- $\left.d_{6}\right): \delta 1.93\left(3 \mathrm{H}, \mathrm{s}, \mathrm{CH}_{3}\right), 2.14\left(3 \mathrm{H}, \mathrm{s}, \mathrm{CH}_{3} \mathrm{Ph}\right), 3.12\left(4 \mathrm{H}, \mathrm{brs}, \mathrm{N}\left(\mathrm{CH}_{2}\right)_{2}\right)$, $3.63\left(4 \mathrm{H}, \mathrm{brs}, \mathrm{N}\left(\mathrm{CH}_{2}\right)_{2}\right), 6.47(1 \mathrm{H}, \mathrm{d}, J=7.9, \mathrm{H} 6), 6.83(1 \mathrm{H}, \mathrm{d}, J=7.9, \mathrm{H} 5), 6.85(1 \mathrm{H}, \mathrm{s}, \mathrm{H} 3) \mathrm{ppm}$.

\subsubsection{Synthesis of 4-(4-Acetylpiperazin-1-yl)-3-methylbenzonitrile 31}

A mixture of $30(1 \mathrm{~mol})$ and $\mathrm{CuCN}(1.4 . \mathrm{mol})$ in NMP was heated at $150{ }^{\circ} \mathrm{C}$ for $4 \mathrm{~h}$. The cool mixture was poured into $3 \%$ aq. $\mathrm{HCl}$ and diluted with ethyl acetate (3 times). The combined organic layers were washed with water ( 3 times), dried over $\mathrm{Na}_{2} \mathrm{SO}_{4}$, and concentrated in vacuo. The residue was triturated with water and collected. White solid, yield $72 \%, \mathrm{~m} . \mathrm{p} . \quad 230-232{ }^{\circ} \mathrm{C}$. MS (EI), $\mathrm{m} / \mathrm{z}$ $\left(I_{\text {relat. }}(\%)\right): 243[\mathrm{M}]^{+}(69)$. Calc. $243.3043, \mathrm{C}_{14} \mathrm{H}_{17} \mathrm{~N}_{3} \mathrm{O} .{ }^{1} \mathrm{H}$ NMR (DMSO- $\left.d_{6}\right): \delta 1.93\left(3 \mathrm{H}, \mathrm{s}, \mathrm{CH}_{3}\right), 2.29$ $\left(3 \mathrm{H}, \mathrm{s}, \mathrm{CH}_{3} \mathrm{Ph}\right), 3.32\left(4 \mathrm{H}, \mathrm{brs}, \mathrm{N}\left(\mathrm{CH}_{2}\right)_{2}\right), 3.63\left(4 \mathrm{H}, \mathrm{brs}, \mathrm{N}\left(\mathrm{CH}_{2}\right)_{2}\right), 6.60(1 \mathrm{H}, \mathrm{d}, J=7.9, \mathrm{H} 6), 7.49(1 \mathrm{H}, \mathrm{d}$, $J=7.9, \mathrm{H} 5), 7.64(1 \mathrm{H}, \mathrm{s}, \mathrm{H} 3) \mathrm{ppm}$.

\subsubsection{Synthesis of 3-(3-Methyl-4-(piperazin-1-yl)phenyl)-5-(trifluoromethyl)-1,2,4-oxadiazole 34}

To a solution of $33(1 \mathrm{mmol})$ in $\mathrm{EtOH}$, concentrated $\mathrm{HCl}(3 \mathrm{~mL})$ was added. The reaction mixture was refluxed for $4 \mathrm{~h}$. The mixture was dissolved in water, neutralized with saturated aq. $\mathrm{NaHCO}_{3}$, and extracted with EtOAc (3 times). The combined organics were dried $\left(\mathrm{Na}_{2} \mathrm{SO}_{4}\right)$ and concentrated in vacuo. The residue was purified by column chromatography (eluent $\left.\mathrm{CHCl}_{3}: \mathrm{MeOH}=10: 1\right), \mathrm{Rf}=0.55$. White solid, yield $65 \%$, m.p. $78-80{ }^{\circ} \mathrm{C}$. MS (EI), $m / z\left(I_{\text {relat. }}(\%)\right): 312[\mathrm{M}]^{+}$(52). Calc. 312.2903, 
$\mathrm{C}_{14} \mathrm{H}_{15} \mathrm{~F}_{3} \mathrm{~N}_{4} \mathrm{O} .{ }^{1} \mathrm{H}$ NMR (DMSO-d $\left.\mathrm{d}_{6}\right): \delta 1.87(1 \mathrm{H}, \mathrm{s}, \mathrm{NH}), 2.23\left(3 \mathrm{H}, \mathrm{s}, \mathrm{CH}_{3} \mathrm{Ph}\right), 2.82\left(4 \mathrm{H}, \mathrm{brs}, \mathrm{N}_{\left(\mathrm{CH}_{2}\right)}\right)$ ), $3.08\left(4 \mathrm{H}, \mathrm{brs}, \mathrm{N}\left(\mathrm{CH}_{2}\right)_{2}\right), 7.00(1 \mathrm{H}, \mathrm{d}, J=7.9, \mathrm{H6}), 7.43(1 \mathrm{H}, \mathrm{d}, J=7.9, \mathrm{H} 5), 7.56(1 \mathrm{H}, \mathrm{s}, \mathrm{H} 3) \mathrm{ppm}$.

\subsubsection{General Procedure for the Synthesis of Compounds 35a,b}

To a solution of $34(1 \mathrm{~mol})$ and DMAP $(1.3 \mathrm{~mol})$ in DCM, the corresponding acid (1 mol) was added in one portion, followed by the addition of EDCI $(1.3 \mathrm{~mol})$ in one portion at rt. The reaction mixture was stirred at rt overnight, after which it was washed successively with $3 \%$ aq. $\mathrm{HCl}$ and water (3 times). The organic layer was dried and concentrated in vacuo. The residue was triturated with water to provide the product as solid and recrystallized from ethanol.

(4-(2-Methyl-4-(5-(trifluoromethyl)-1,2,4-oxadiazol-3-yl)phenyl)piperazin-1-yl)(3-phenylisoxazol-5yl)methanone 35a, White solid, yield $44 \%$, m.p. $134-136{ }^{\circ} \mathrm{C}$. MS (EI), $m / z\left(I_{\text {relat }}(\%)\right): 483[\mathrm{M}]^{+}$ (52). Calc. 483.4425, $\mathrm{C}_{24} \mathrm{H}_{20} \mathrm{~F}_{3} \mathrm{~N}_{5} \mathrm{O}_{3} .{ }^{1} \mathrm{H}-\mathrm{NMR}$ (DMSO-d $\left.d_{6}\right): \delta 2.39\left(3 \mathrm{H}, \mathrm{s}, \mathrm{CH}_{3} \mathrm{Ph}\right), 3.05(4 \mathrm{H}, \mathrm{m}$, $\left.\mathrm{N}\left(\mathrm{CH}_{2}\right)_{2}\right), 3.84\left(4 \mathrm{H}\right.$, brt, $\left.\mathrm{CON}\left(\mathrm{CH}_{2}\right)_{2}\right), 4.37\left(2 \mathrm{H}\right.$, brs, $\left.\mathrm{NCH}_{2}\right), 7.24(1 \mathrm{H}$, д, $J=8.5, \mathrm{H} 6), 7.55(3 \mathrm{H}, \mathrm{m}$, $\mathrm{m}, \mathrm{p}-\mathrm{Ph}), 7.59(1 \mathrm{H}, \mathrm{s}$, isoxazole), $7.86(1 \mathrm{H}, \mathrm{d}, J=8.5, \mathrm{H} 5), 7.87(1 \mathrm{H}, \mathrm{s}, \mathrm{H} 3), 7.96(2 \mathrm{H}, \mathrm{m}, \mathrm{o}-\mathrm{Ph}) \mathrm{ppm}$. ${ }^{13} \mathrm{C}-\mathrm{NMR}$ (DMSO- $d_{6}$ ): $\delta 17.68,46.67,46.67,50.24,50.24,100.78,115.90(\mathrm{q}, J=273.2), 118.51,120.05$, $127.10,127.10,127.32,128.17,128.70,128.75,129.71,129.71,130.45,150.10,155.66,159.67,162.39,166.10$ $(\mathrm{q}, J=43.3), 167.93 \mathrm{ppm}$.

(5-Methyl-3-phenylisoxazol-4-yl)(4-(2-methyl-4-(5-(trifluoromethyl)-1,2,4-oxadiazol-3-yl)phenyl)pi-perazin1-yl)methanone 35b, White solid, yield 38\%, m.p. $119-121^{\circ} \mathrm{C}$. MS (EI), $m / z$ (I Irelat. $\left.(\%)\right): 497[\mathrm{M}]^{+}(64)$. Calc. 497.4691, $\mathrm{C}_{25} \mathrm{H}_{22} \mathrm{~F}_{3} \mathrm{~N}_{5} \mathrm{O}_{3} .{ }^{1} \mathrm{H}-\mathrm{NMR}\left(\mathrm{CDCl}_{3}\right): \delta 2.39\left(3 \mathrm{H}, \mathrm{s}, \mathrm{CH}_{3} \mathrm{Ph}\right), 2.57\left(3 \mathrm{H}, \mathrm{s}, \mathrm{CH}_{3}\right), 2.87-4.07$ $\left(8 \mathrm{H}, \mathrm{m}, \mathrm{N}\left(\mathrm{CH}_{2}\right)_{2}\right), 6.92(1 \mathrm{H}, \mathrm{d}, J=7.86, \mathrm{H} 6), 7.51(3 \mathrm{H}, \mathrm{m}, \mathrm{m}, \mathrm{p}-\mathrm{Ph}), 7.52(1 \mathrm{H}, \mathrm{s}$, isoxazole $), 7.70(2 \mathrm{H}, \mathrm{m}$, o-Ph), $7.92(1 \mathrm{H}, \mathrm{d}, J=8.5, \mathrm{H} 5), 7.94(1 \mathrm{H}, \mathrm{s}, \mathrm{H} 3) \mathrm{ppm} .{ }^{13} \mathrm{C}-\mathrm{NMR}\left(\mathrm{DMSO}_{\mathrm{d}}\right.$ ): $\delta 12.40,17.94,46.75,46.75$, 50.32, 50.32, 112.0, 115.70 (q, $J=273.4), 118.43,120.00,127.12,128.42,128.65,128.89,129.72,129.72$, $130.00,130.00,130.67,149.74,161.87,164.00,166.02(\mathrm{q}, J=43.4), 167.31,169.98 \mathrm{ppm}$.

3.2.16. Synthesis of 3-(3-Methyl-4-(4-(prop-2-yn-1-yl)piperazin-1-yl)phenyl)-5-(trifluoromethyl)1,2,4-oxa-diazole 37

A mixture of $34(1 \mathrm{mmol})$, propargyl bromide $(1.3 \mathrm{mmol})$, finely divided $\mathrm{K}_{2} \mathrm{CO}_{3}(3 \mathrm{mmol})$, and $\mathrm{KI}$ $(0.1 \mathrm{mmol})$ in acetonitrile was heated at $50^{\circ} \mathrm{C}$ for $2 \mathrm{~h}$. The hot mixture was filtered, and the remaining solids were washed with acetonitrile. The combined organic filtrates were concentrated in vacuo. The residue was triturated with water, and precipitate was collected. Yellow solid, yield 77\%, m.p. 75-78 ${ }^{\circ} \mathrm{C}$. MS (EI), $m / z\left(I_{\text {relat. }}(\%)\right): 350[\mathrm{M}]^{+}(71)$. Calc. $350.3383, \mathrm{C}_{17} \mathrm{H}_{17} \mathrm{~F}_{3} \mathrm{~N}_{4} \mathrm{O} .{ }^{1} \mathrm{H}-\mathrm{NMR}\left(\mathrm{DMSO}-d_{6}\right): \delta$ $2.23\left(3 \mathrm{H}, \mathrm{s}, \mathrm{CH}_{3} \mathrm{Ph}\right), 2.54\left(4 \mathrm{H}, \mathrm{brs}, \mathrm{N}\left(\mathrm{CH}_{2}\right)_{2}\right), 2.79\left(1 \mathrm{H}, \mathrm{s}, \mathrm{CHCCH}_{2}\right), 3.12\left(4 \mathrm{H}, \mathrm{brs}, \mathrm{N}\left(\mathrm{CH}_{2}\right)_{2}\right), 3.33(2 \mathrm{H}$, $\left.\mathrm{s}, \mathrm{NCH}_{2}\right), 7.00(1 \mathrm{H}, \mathrm{d}, J=7.9, \mathrm{H} 6), 7.43(1 \mathrm{H}, \mathrm{d}, J=7.9, \mathrm{H} 5), 7.56(1 \mathrm{H}, \mathrm{s}, \mathrm{H} 3) \mathrm{ppm}$.

\subsection{Antiviral Testing of the Compounds}

\subsubsection{Viruses and Cells}

Influenza A virus (strain A/Puerto Rico/8/1934 H1N1) and coxsackievirus 3 (strain Nancy) were obtained from the collection of viruses of the Pasteur Institute (St. Petersburg, Russia). Prior to the experiment, influenza A virus (IAV) and coxsackievirus 3 (CVB3) were grown in MDCK (ATCC \# PTA-6500) and Vero cells (ATCC \#CCL-81), respectively, for three days at $37{ }^{\circ} \mathrm{C}$ and $5 \% \mathrm{CO}_{2}$. Infectious titers of IAV and CVB3 (in TCID 50 ) were determined in MDCK and Vero cells, respectively, by endpoint dilution assay using the following procedure. Cells were seeded into 96-wells plates in Eagles minimal essential medium (MEM) supplemented with $10 \%$ fetal bovine serum (FBS). After $24 \mathrm{~h}$, the media was aspirated, the wells were washed with saline, and serial tenfold dilutions of virus stock were added (100 $\mu \mathrm{L}$ per well) in duplicates. The plates were incubated at $+4{ }^{\circ} \mathrm{C}$ for $1 \mathrm{~h}$, then unbounded virus was discarded, and fresh MEM without FBS was added to the wells $(200 \mu \mathrm{L}$ per 
well). The plates were incubated at $37^{\circ} \mathrm{C}$ in $5 \% \mathrm{CO}_{2}$ and observed daily for cytopathic effect (CPE). After $72 \mathrm{~h}$, the viral titer was calculated in $\mathrm{TCID}_{50}$ using the method of Reed and Muench.

\subsubsection{Cytotoxicity Assay}

The microtetrazolium test (MTT) was used to study the cytotoxicity of the compounds [24]. The experiment was repeated three times. Vero cells were seeded in 96-well plates in Eagles minimal essential medium (MEM) supplemented with $10 \%$ FBS. After $24 \mathrm{~h}$, the media was removed, and the wells were washed with saline. Compounds were dissolved in DMSO, and a series of three-fold dilutions of each compound and pleconaril (1000-4 $\mu \mathrm{g} / \mathrm{mL})$ in MEM without FBS were prepared and added to the cells in quadriplicates ( $200 \mu \mathrm{L}$ per well). The maximal concentration of DMSO was $0.5 \%$ MEM with $0.5 \%$ DMSO and was added to cell control wells. Cells were incubated for $24 \mathrm{~h}$ at $37^{\circ} \mathrm{C}$ in $5 \% \mathrm{CO}_{2}$ in the presence of the dissolved compounds. The cells were then washed with saline, and a solution of 3-(4,5-dimethylthiazolyl-2) 2,5-diphenyltetrazolium bromide (ICN Biochemicals Inc., Aurora, OH, USA) $(0.5 \mu \mathrm{g} / \mathrm{mL})$ in MEM was added to the wells $(100 \mu \mathrm{L}$ per well). After $2 \mathrm{~h}$ of incubation at $37^{\circ} \mathrm{C}$ in $5 \% \mathrm{CO}_{2}$, the supernatant from wells was discarded, and the formazan residue was dissolved in DMSO (100 $\mu \mathrm{L}$ per well). The optical density of cells was then measured on a Victor 21,440 multifunctional reader (Perkin Elmer, Turku, Finland) at a wavelength of $535 \mathrm{~nm}$ and plotted against the concentration of the compounds to generate the dose-response curve. The $50 \%$ cytotoxic dose $\left(\mathrm{CC}_{50}\right)$ of each compound (i.e., the compound concentration that causes the death of $50 \%$ of cells in a culture, or decreases the optical density twice as compared to the control wells) was calculated using four-parameter logistic nonlinear regression model. For some compounds ( $35 \mathbf{a}$ and $\mathbf{3 5 b}$ ) cytotoxicity towards MDCK cell line was determined using the procedure above, but the cells were exposed to compounds for $72 \mathrm{~h}$.

\subsubsection{Antiviral Activity Determination}

Antiviral activity of the compound against CVB3 was evaluated using viral yield reduction assay. The experiment was repeated three times. Vero cells were seeded in MEM supplemented with $5 \%$ FBS in 24-well plates. When the cells confluence reached $100 \%$, giving an approximate cell density of $0.2 \times 10^{6}$ per well, the compounds were dissolved in DMSO, and a series of three-fold non-toxic dilutions of each compound $(600-6 \mu \mathrm{g} / \mathrm{mL})$ and pleconaril $(600-0.6 \mu \mathrm{g} / \mathrm{mL})$ in MEM without FBS was prepared, added to the cells (500 $\mu \mathrm{L}$ per well), and incubated at $37^{\circ} \mathrm{C}$ in $5 \% \mathrm{CO}_{2}$. After $1 \mathrm{~h}$, viral suspension in MEM without FBS was added to the all wells at MOI 0.01 (500 $\mu \mathrm{L}$ per well) except cell control, and the plates were incubated at $4{ }^{\circ} \mathrm{C}$ for $1 \mathrm{~h}$. Thereafter, the cell supernatant was removed, and MEM without FBS was added to all wells ( $1 \mathrm{~mL}$ ). After $24 \mathrm{~h}$ of incubation at $37^{\circ} \mathrm{C}$ in $5 \% \mathrm{CO}_{2}$, the viral progeny infectious titers (in $\mathrm{TCID}_{50}$ ) for each compound concentration, cell control, and virus control wells were determined in Vero cells by endpoint dilution assay. The supernatants from corresponding wells of 24-plates were serially diluted in titer tubes and added to $96-$ well plates in duplicates (200 $\mu \mathrm{L}$ per well). The plates were incubated at $37{ }^{\circ} \mathrm{C}$ in $5 \% \mathrm{CO}_{2}$ and observed daily for cytopathic effect. After $72 \mathrm{~h}$, the viral titer in each compound concentration, cell control, and virus control wells was calculated in TCID 50 using the method of Reed and Muench. The infectious titer of virus progeny was plotted against the concentration of the compounds to generate the dose-response curve. The $50 \%$ inhibition concentration $\left(\mathrm{IC}_{50}\right)$ of each compound tested (i.e., the compound concentration that decreases the infectious viral progeny titer twice as compared to the control wells) was calculated using four-parameter logistic nonlinear regression model. Selectivity index (SI) was calculated for each compound tested as a ratio of $\mathrm{CC}_{50}$ to $\mathrm{IC}_{50}$ values.

Antiviral activity of the compound against IAV was evaluated using hemagglutination test. The experiment was repeated three times. MDCK cells were seeded in MEM supplemented with 5\% FBS in 96-well plates. When the cells confluence reached $100 \%$, the plate was washed with saline, the tested compounds were dissolved in DMSO, and a series of three-fold non-toxic dilutions of each compound $(600-6 \mu \mathrm{g} / \mathrm{mL})$ in MEM without FBS was prepared and added to the cells (100 $\mu \mathrm{L}$ per well). 
Tenfold dilutions of viral suspension in MEM without FBS were added to all wells (100 $\mu \mathrm{L}$ per well) except cell control, and the plates were incubated at $37^{\circ} \mathrm{C}$ for $72 \mathrm{~h}$. Thereafter, the cell supernatant $(100 \mu \mathrm{L})$ was transferred to a "V bottom" 96-well microtiter plate and mixed with $100 \mu \mathrm{L}$ of $1 \%$ chicken erythrocytes (RBC). After $1 \mathrm{~h}$, cell control was checked for complete settling of RBCs, and the viral titer was determined. The $50 \%$ inhibitory concentration $\left(\mathrm{IC}_{50}\right)$ of each compound tested (i.e., the compound concentration that decreases the viral titer twice as compared to the control wells) was calculated using four-parameter logistic nonlinear regression model. Selectivity index (SI) was calculated for each compound tested as a ratio of $\mathrm{CC}_{50}$ to $\mathrm{IC}_{50}$ values.

\section{Conclusions}

We have synthesized a series of novel pleconaril-based compounds with modified O-alkyl linker. All the derivatives were characterized by their MS and NMR data. Synthesized target compounds were evaluated for their in vitro antiviral activity against coxsackievirus B3 strain Nancy. Among these compounds, 21 with an $\mathrm{IC}_{50}$ value of $6.8 \mu \mathrm{M}$ and SI of 248 , is the most active anticoxsackievirus agent compared to other studied compounds (including pleconaril) with low cytotoxicity. The results of this study demonstrate the possibility to further improve pleconaril and evolve it to develop novel potent selective anti-coxsackievirus inhibitors that have activity against the B3 Nancy strain.

Author Contributions: Conceptualization, V.M.; synthesis and characterization of the compounds, A.E.; biological testing of the compounds, A.V. and A.G.; writing-original draft preparation, A.E. and V.M.; writing-review and editing, S.E. and V.Z. All authors have read and agreed to the published version of the manuscript.

Funding: RF State Contract 0104-2019-0012 funded this research (synthetic part).

Conflicts of Interest: The authors declare no conflict of interest.

\section{References}

1. Lugo, D.; Krogstad, P. Enteroviruses in the Early 21st Century: New Manifestations and Challenges. Curr. Opin Pediatr. 2016, 28, 107-113. [CrossRef] [PubMed]

2. Gaaloul, I.; Riabi, S.; Harrath, R.; Hunter, T.; Hamda, K.B.; Ghzala, A.B.; Huber, S.; Aouni, M. Coxsackievirus $\mathrm{B}$ detection in cases of myocarditis, myopericarditis, pericarditis and dilated cardiomyopathy in hospitalized patients. Mol. Med. Rep. 2014, 10, 2811-2818. [CrossRef] [PubMed]

3. Garmaroudi, F.S.; Marchant, D.; Hendry, R.; Luo, H.; Yang, D.; Ye, X.; Shi, J.; McManus, B.M. Coxsackievirus B3 replication and pathogenesis. Future Microbiol. 2015, 10, 629-653. [CrossRef]

4. Brunetti, L.; DeSantis, E.R. Treatment of viral myocarditis caused by coxsackievirus B. Am. J. Health Syst. Pharm. 2008, 65, 132-137. [CrossRef]

5. Pollack, A.; Kontorovich, A.R.; Fuster, V.; Dec, G.W. Viral myocarditis - diagnosis, treatment options, and current controversies. Nat. Rev. Cardiol. 2015, 12, 670-680. [CrossRef]

6. Baggen, J.; Thibaut, H.J.; Strating, J.R.P.M.; van Kuppeveld, F.J.M. The life cycle of non-polio enteroviruses and how to target it. Nat. Rev. Microbiol. 2018, 16, 368-381. [CrossRef]

7. Groarke, J.M.; Pevear, D.C. Attenuated virulence of pleconaril-resistant coxsackievirus B3 variants. J. Infect. Dis. 1999, 179, 1538-1541. [CrossRef]

8. Pevear, D.C.; Tull, T.M.; Seipel, M.E.; Groarke, J.M. Activity of Pleconaril against Enteroviruses. Antimicrob. Agents Chemother. 1999, 43, 2109-2115. [CrossRef]

9. Schmidtke, M.; Hammerschmidt, E.; Schüler, S.; Zell, R.; Birch-Hirschfeld, E.; Makarov, V.A.; Riabova, O.B.; Wutzler, P. Susceptibility of coxsackievirus B3 laboratory strains and clinical isolates to the capsid function inhibitor pleconaril: Antiviral studies with virus chimeras demonstrate the crucial role of amino acid 1092 in treatment. J. Antimicrob. Chemother. 2005, 56, 648-656. [CrossRef]

10. Schmidtke, M.; Wutzler, P.; Zieger, R.; Riabova, O.B.; Makarov, V.A. New pleconaril and [(biphenyloxy)propyl]isoxazole derivatives with substitutions in the central ring exhibit antiviral activity against pleconaril-resistant coxsackievirus B3. Antivir. Res. 2009, 81, 56-63. [CrossRef] 
11. Egorova, A.; Kazakova, E.; Jahn, B.; Ekins, S.; Makarov, V.; Schmidtke, M. Novel pleconaril derivatives: Influence of substituents in the isoxazole and phenyl rings on the antiviral activity against enteroviruses. Eur. J. Med. Chem. 2020, epub12007. [CrossRef]

12. Diana, G.D.; McKinlay, M.A.; Otto, M.J.; Akullian, V.; Oglesby, C. (4,5-Dihydro-2-oxazolyl)phenoxyalkylisoxazoles. Inhibitors of picornavirus uncoating. J. Med. Chem. 1985, 28, 1906-1910. [CrossRef]

13. Diana, G.D.; Oglesby, R.C.; Akullian, V.; Carabateas, P.M.; Cutcliffe, D.; Mallamo, J.P.; Otto, M.J.; McKinlay, M.A.; Maliski, E.G.; Michalec, S.J. Structure-activity studies of 5-[[4-(4,5-dihydro-2-oxazolyl)phenoxy]alkyl]-3-methylisoxazoles: Inhibitors of picornavirus uncoating. J. Med. Chem. 1987, 30, 383-388. [CrossRef]

14. Friedman, L.; Shechter, H. Dimethylformamide as a Useful Solvent in Preparing Nitriles from Aryl Halides and Cuprous Cyanide; Improved Isolation Techniques. J. Org. Chem. 1961, 26, 2522-2524. [CrossRef]

15. Cheng, H.; Wan, J.; Lin, M.I.; Liu, Y.; Lu, X.; Liu, J.; Xu, Y.; Chen, J.; Tu, Z.; Cheng, Y.S.; et al. Design, synthesis, and in vitro biological evaluation of $1 \mathrm{H}-1,2,3$-triazole-4-carboxamide derivatives as new anti-influenza A agents targeting virus nucleoprotein. J. Med. Chem. 2012, 55, 2144-2153. [CrossRef]

16. Newman, M.S.; Hetzel, F.W. Thiophenols from phenols: 2-naphthalenethiol. Org. Synth. 1971, 51, 139. [CrossRef]

17. Zysman-Colman, E.; Arias, K.; Siegel, J.S. Synthesis of arylbromides from arenes and N-bromosuccinimide (NBS) in acetonitrile-A convenient method for aromatic bromination. Can. J. Chem. 2009, 87, 440-447. [CrossRef]

18. Gu, T.; Rachwal, B.; Siddiqui, O.; Rachwal, S.; Kitahara, I.; Simavoryan, S.; Wang, P.; Yamamoto, M. Chromophores for photochromic compositions useful for three dimensional display applications. U.S. patent 2017/0044373 A1, 29 October 2015.

19. Liu, K.G.; Robichaud, A.J. A general and convenient synthesis of N-aryl piperazines. Tetrahedron Lett. 2005, 46, 7921-7922. [CrossRef]

20. Ge, Z.; Ji, Q.; Chen, C.; Liao, Q.; Wu, H.; Liu, X.; Huang, Y.; Yuan, L.; Liao, F. Synthesis and biological evaluation of novel 3-substituted amino-4-hydroxylcoumarin derivatives as chitin synthase inhibitors and antifungal agents. J. Enzyme Inhib. Med. Chem. 2016, 31, 219-228. [CrossRef]

21. Kozikowski, A.P.; Adamcz, M. Methods for the stereoselective cis-cyanohydroxylation and -carboxyhydroxylation of olefins. J. Org. Chem. 1983, 48, 366-372. [CrossRef]

22. Piotrowski, D.W.; Rogers, B.N.; McWhorter, W.W.; Walker, D.P.; Corbett, J.W.; Groppi, V.E.; Rudmann, D.G. Positive Allosteric Modulators of the Nicotinic Acetylcholine Receptor. U.S. Patent 2003/0236287 A1, 25 December 2003.

23. Li, W.T.; Hwang, D.R.; Chen, C.P.; Shen, C.W.; Huang, C.L.; Chen, T.W.; Lin, C.H.; Chang, Y.L.; Chang, Y.Y.; Lo, Y.K.; et al. Synthesis and biological evaluation of N-heterocyclic indolyl glyoxylamides as orally active anticancer agents. J. Med. Chem. 2003, 46, 1706-1715. [CrossRef]

24. Mosmann, T. Rapid colorimetric assay for cellular growth and survival: Application to proliferation and cytotoxicity assays. J. Immunol. Methods. 1983, 65, 55-63. [CrossRef]

Sample Availability: Not available.

(C) 2020 by the authors. Licensee MDPI, Basel, Switzerland. This article is an open access article distributed under the terms and conditions of the Creative Commons Attribution (CC BY) license (http://creativecommons.org/licenses/by/4.0/). 